\title{
On Efficient Recommendations for Online Exchange
} Markets

\author{
by \\ Zeinab Abbassi
}

B.Sc., Sharif University of Technology, 2006

A THESIS SUBMITTED IN PARTIAL FULFILLMENT

OF THE REQUIREMENTS FOR THE DEGREE OF

Master of Science

in

THE FACULTY OF GRADUATE STUDIES

(Computer Science)

The University Of British Columbia

(Vancouver)

August 2008

(C) Zeinab Abbassi 


\section{Abstract}

Motivated by the popularity of marketplace applications over social networks, we study optimal recommendation algorithms for online exchange markets. Examples of such markets include peerflix.com and readitswapit.co.uk. We model these markets as a social network in which each user has two associated lists: The item list, i.e, the set of items the user is willing to give away, and the wish list, i.e., the set of items the user is interested in receiving. A transaction involves a user giving an item to another user. Users are motivated to engage in transactions in expectation of realizing their wishes. Wishes may be realized by a pair of users swapping items corresponding to each other's wishes, but more generally by means of users exchanging items through a cycle, where each user gives an item to the next user in a cycle, in accordance with the receiving user's wishes.

In this thesis, we first consider the problem of how to efficiently generate recommendations for item exchange cycles in an online market social network. We consider deterministic and probabilistic models and show that under both models, the problem of determining an optimal set of recommendations that maximizes the expected value of items exchanged is NP-hard and develop efficient approximation algorithms for both models. Next, we study exchange markets over time and try to optimize users' waiting times, 
Abstract

and fairness where by fairness we mean: give higher priority to users who contribute more to the system in addition to maximizing expected value. We show that by introducing the concept of points, average waiting time can be improved by a large factor. By designing a credit system, we try to maximize fairness in the system. We show not only is the fairness optimization problem NP-hard, but also inapproximable within any multiplicative factor. We propose two heuristic algorithms, one of which is based on rounding the solution to a linear programming relaxation and the other is a greedy algorithm. For both the one-shot market and the overtime market studied in this thesis, we conduct a comprehensive set of experiments, and explore the performance and also scalability of the proposed algorithms. Our experiments suggest that the performance of our algorithms in practice could be much better than the worst-case performance guarantee factors. 


\section{Table of Contents}

Abstract $\ldots \ldots \ldots \ldots \ldots \ldots \ldots$ ii

Table of Contents $\ldots \ldots \ldots \ldots \ldots \ldots$ iv

List of Tables $\ldots \ldots \ldots \ldots \ldots \ldots$ vii

List of Figures $\ldots \ldots \ldots \ldots \ldots$ viii

Acknowledgements $\ldots \ldots \ldots \ldots \ldots \ldots$ xi

1 Introduction $\ldots \ldots \ldots \ldots \ldots \ldots \ldots$

1.1 Structure of the Thesis $\ldots \ldots \ldots \ldots 7$

2 One-shot Exchange Markets .............. 8

2.1 Related Work .................. 9

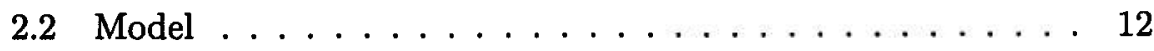

2.3 Hardness Results . . . . . . . . . . . . . . . . 19

2.4 The Algorithms $\ldots \ldots \ldots \ldots \ldots \ldots$

2.4.1 Algorithm Maximal . . . . . . . . . . 27

2.4 .2 Greedy Algorithm . . . . . . . . . . . . 29

2.4 .3 Local Search Algorithm . . . . . . . . . . . . . 30 
2.4.4 Greedy/Local Search . . . . . . . . . . 30

2.5 Analysis $\ldots \ldots \ldots \ldots \ldots \ldots \ldots \ldots \ldots$

2.5.1 Performance ................. 31

2.5 .2 Running Time . . . . . . . . . . . . . 34

2.6 Experimental Results $\ldots \ldots \ldots \ldots \ldots$

2.6.1 Data Set . . . . . . . . . . . . 35

2.6 .2 Experiments $\ldots \ldots \ldots \ldots$

2.7 Conclusion $\ldots \ldots \ldots \ldots \ldots \ldots \ldots \ldots$

3 Online Exchange Markets Over Time . . . . . . . . . 52

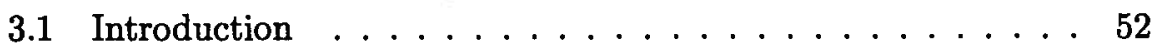

3.2 Related Work . . . . . . . . . . . . 53

3.2.1 Scheduling Problems $\ldots \ldots \ldots \ldots$

3.2.2 Fairness Maximization in P2P Systems ...... 55

3.3 Model . . . . . . . . . . . . . . . 56

3.4 Minimizing Average Waiting Time $\ldots \ldots \ldots \ldots$

3.5 A Credit System $\ldots \ldots \ldots \ldots$

3.6 Maximizing Fairness $\ldots \ldots \ldots$. . . . . . . . . 62

3.7 A Linear Programming Formulation . . . . . . . . . . 64

3.8 A Strong Inapproximability Result . . . . . . . . . . 66

3.9 Heuristic Algorithms $\ldots \ldots \ldots 68$

3.9.1 Greedy Algorithm . . . . . . . . . . . . 68

3.9.2 An LP-rounding Algorithm . . . . . . . . . . . 69

3.10 Experimental Evaluation $\ldots \ldots \ldots \ldots$

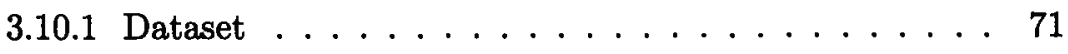


Table of Contents

3.10 .2 Experiments $\ldots \ldots \ldots \ldots \ldots \ldots \ldots \ldots$
3.11 Conclusion $\ldots \ldots \ldots \ldots \ldots \ldots$

4 Conclusions and Future Work $\ldots \ldots \ldots$

Bibliography .................... 82 


\section{List of Tables}

2.1 Alice's Item and Wish list . . . . . . . . . . . . 13

2.2 Bob's Item and Wish list $\ldots \ldots \ldots \ldots \ldots$

2.3 Joe's Item and Wish list . . . . . . . . . . . . . 14

2.4 Amy's Item and Wish list $\ldots \ldots \ldots \ldots$

2.5 Mary's Item and Wish list . . . . . . . . . . 15

$2.6 \%$ Increase in coverage when considering cycles of length 3,4

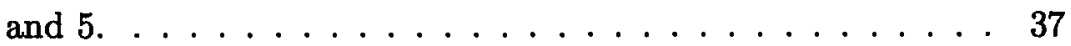




\section{List of Figures}

2.1 Both exchanges cannot be present in the system at the same time. . . . . . . . . . . . . . . 16

2.2 Exchange cycle of length three: [(Alice, $\left.B_{7}\right)$, (Bob, $\left.B_{4}\right)$, (Amy,

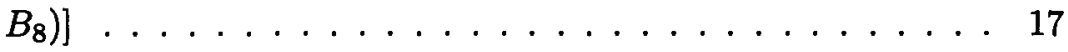

2.3 Hardness Proof . . . . . . . . . . . . . . . . . 24

2.4 Graph Representation of Running Example. . . . . . . . . . 28

2.5 Description of the Maximal Algorithm . . . . . . . . . . 41

2.6 Description of the Greedy Algorithm . . . . . . . . . 42

2.7 Description of the Local Search Algorithm . . . . . . . . . 43

2.8 Results of the Maximal algorithm on datasets of size 10000, 25000 and $50000 \ldots \ldots \ldots \ldots \ldots \ldots \ldots$

2.9 Results of the Greedy algorithm on datasets of size 10000, 25000 and $50000 \ldots \ldots \ldots \ldots \ldots \ldots$

2.10 Results of the Local Search algorithm on datasets of size 10000,25000 and $50000 \ldots \ldots \ldots \ldots$

2.11 Percentage of coverage for different $M$ 's in the Maximal algorithm. . . . . . . . . . . . . . 46

2.12 Average Running Time of Various Algorithms. . . . . . . . . 47 
2.13 Running Time of Algorithm Maximal on dataset of size 10,0000

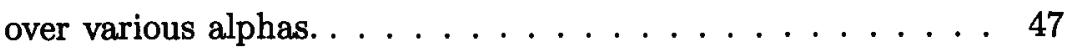

2.14 Running Time of Algorithm Maximal on dataset of size 25,0000 over various alphas. . . . . . . . . . . . . . 48

2.15 Running Time of Algorithm Maximal on dataset of size 50,0000 over various alphas. . . . . . . . . . . . . . 48

2.16 Running Time of Algorithm Greedy on dataset of size 10,0000 over various alphas. . . . . . . . . . . . . . . 49

2.17 Running Time of Algorithm Greedy on dataset of size 25,0000 over various alphas. . . . . . . . . . . . . . 49

2.18 Running Time of Algorithm Greedy on dataset of size 50,0000 over various alphas. . . . . . . . . . . . . 50

2.19 Running Time of Algorithm Local Search on dataset of size 10,0000 over various alphas. . . . . . . . . . . 50

2.20 Running Time of Algorithm Maximal on dataset of size 25,0000 over various alphas. . . . . . . . . . . . 51

2.21 Running Time of Algorithm Maximal on dataset of size 50,0000 over various alphas. . . . . . . . . . . . 51

3.1 Performance of the heuristics compared to the optimal solution given by ILP on dataset of 10 users. . . . . . . . . . 72

3.2 Performance of the heuristics compared to the optimal solution given by ILP on dataset of 20 users. . . . . . . . . . 73

3.3 Performance of different algorithms on dataset of size 100. . 74

3.4 Performance of different algorithms on dataset of size 500. . 75 


\section{List of Figures}

3.5 Performance of different algorithms on dataset of size 1000. . 76

3.6 Running times for dataset of size $100 \ldots \ldots \ldots \ldots$

3.7 Running times for dataset of size $500 \ldots \ldots \ldots \ldots$

3.8 Running times for dataset of size $1000 \ldots \ldots \ldots 77$

3.9 Number of transacting users in 6 consecutive time windows. . 78 


\section{Acknowledgements}

I would like to take this opportunity to thank my supervisor, Professor Laks Lakshmanan, for his great help and guidance through this thesis. I am indebted to him for his insightful comments on my research and his support during the past year.

I am also grateful to Professor Raymond $\mathrm{Ng}$ for reviewing my thesis and for his valuable comments.

I owe thanks to all members of the Database Management and Mining lab, and also Holly Kwan for making my years at UBC memorable.

Last, but not least, I would like to express my gratitude to my dear family, my parents, Laya and Abbas; my brother and his wife, Hossein and Sara; and my husband Vahab, whose endless love and support has always been a bliss for me. Thanks for everything. 


\section{Chapter 1}

\section{Introduction}

Sociologists have studied social networks extensively in the past years. The six degrees of separation experiment performed by Stanley Milgram in the late 1960s is a well-known and also remarkable instance of such studies. The networks studied in social sciences are typically small due to the fact that collecting information is difficult and typically done by the circulation of questionnaires or doing interviews, asking respondents to detail their interactions with others [29]. These days, the availability of the Internet has caused dramatic changes. Online social networks have emerged and become popular, data is available in a much larger scale than before, and the increased computational power lets us study the statistical properties of the graph in addition to the individual studies done before. The field of social networks in computer science is rapidly growing due to the relatively recent popularity of online social networks such as MySpace and FaceBook. Users are spending increasing amounts of time on social network websites. For instance, a recent survey [1] that ranks websites based on average time spent by a user, identifies MySpace and Facebook among the top 10 websites, and MySpace stands at the top by $11.9 \%$.

On the other hand, the increasing amount of choices available on the 
Web has made it increasingly difficult to find what one is looking for. Three methods are commonly applied to help web users in locating their desired items: search engines, taxonomies and, more recently, recommender systems [24]. This is where recommender systems come into the play. Recommender systems have been extensively studied. However, in most of them the properties of social networks are ignored.

One important example of social interaction among users on the Internet is online exchange markets which are currently present on the web. Some examples are as follows:

- peerflix.com [3] for exchanging movies: It lets the users exchange their DVDs with each other instead of renting them.

- readitswapit.co.uk [4] allows book lovers to exchange their already read books and receive new books in return. Almost all of the "matching" (i.e., finding books and owners to exchange with) is done manually by the user herself, meaning that she has to go and find her desired book in a library and then mark it. The owner of the desired book will be informed by an email and will check the seeker's list of books and if willing to do the exchange, they will post the books for each other.

- oddshoe.org: [2] is a national organization which is a resource for new, quality single shoes and pairs of significantly different sizes. Many have this need due to injury, disease and genetic disorders. This organization has been around since 1943 and the exchanges are not done online. 
- Intervac International is a world-wide non-profit organization which has facilitated home exchanges between its members since 1953. They operate a multi-language real-time database of home exchange listings on their website with an option to receive printed catalogues of listings.

- Joebarter.com is an online barter site, plus an array of networking services and resources to pay the typical charges and fees associated with online barter exchanges.

Other than the above examples for exchange markets, various marketplaces for exchanging items can be designed as new applications over online social networks on the web. This can be already seen to be happening over Facebook.

Motivated by the above, we study optimal "matching" algorithms for online exchange markets. Here, by "matching", we mean finding a set of users and items such that they can exchange items in a cycle of length two or more. ${ }^{1}$ A main motivation behind our work is the lack of comprehensive and efficient recommendation algorithms for item exchange in the current systems. These algorithms can improve the quality of user experience in exchange markets, and in turn, they can make various ways of monetizing such systems, such as online advertising, more effective.

In Chapter 2 we focus on the problem of generating recommendations under two models: deterministic model and probabilistic model. In both models each user receives an item in return for the item which she gives away. We formulate the problem of finding recommendations as that of

\footnotetext{
${ }^{1}$ No confusion should arise with the standard graph-theoretic notion of matching. The context will make the meaning clear.
} 
finding a set of conflict-free cycles in the graph. Conflict-free means cycles in the set are pairwise edge-disjoint, a condition necessary to ensure that when a user commits to a recommended exchange, she is not surprised that the recommended item is already taken. Thus, a recommendation consists of a set of conflict-free cycles. The value of a recommendation is the number of items (potentially) exchanged through the cycles recommended. When the cycle length is restricted to two, we call the corresponding problem simple exchange markets. In a simple exchange market, the only acceptable recommendation is a set of (conflict-free) swaps, called a swap recommendation. We prove the surprising result that even finding an optimal swap recommendation is NP-complete.

Next, we consider a more general situation where exchange through short cycles, i.e., cycles of length up to $k$, where $k$ is a small predetermined constant. We typically consider $k=2, \ldots, 5$. We call this exchange markets with short cycles. The reason why we focus on short cycles is that, even if one user in the cycle withdraws the transaction, the whole exchange cycle collapses. If we have long cycles, then more users would be affected. Now, a recommendation is a set of conflict-free cycles with length bounded by $k$. Of course, the hardness extends to finding optimal recommendations for this case.

Finally, we consider the probabilistic exchange market. In this case, there is a probability associated with each user engaging in a transaction with any other user. As well, there is a probability of a user being willing to trade one item for another. We assume all probabilities are independent. A recommendation is defined in exactly the same way as for exchange mar- 
kets through short cycles. The value of a recommendation is the expected number of items exchanged. The contribution of a cycle to the value of a recommendation is the number of items exchanged in the cycle multiplied by the product of all probabilities associated with the cycle. We show that finding an optimal recommendation remains NP-complete.

We develop heuristic and approximation algorithms for finding optimal recommendation for all three kinds of markets. We discuss three approximation algorithms - Greedy, Local search, and combination of greedy and local search - and one heuristic algorithm - Maximal. We prove that the approximation algorithms find recommendations that are within a factor of $2 k, 2 k-1$, and $(2 k+1) / 3$ of the optimal, respectively. We also analyze the complexity of the proposed algorithms. While Maximal has no provable approximation guarantees, it is by far the most efficient.

We conduct a detailed empirical study of all algorithms proposed using synthetic data that we generated. The data was generated to conform to power law distributions commonly found in social networks. Our experiments show that even though Maximal has no theoretical approximation guarantees, recommendations found by Maximal are very competitive with those found by the approximation algorithms. On the other hand, Maximal significantly outperforms the other algorithms when it comes to scalability w.r.t. network size and the sizes of item and wish lists.

In chapter 23 we consider exchange markets over time. We set new objectives in this new setting and try to achieve them by introducing efficient features and algorithms. Our objectives are: 
- Maximizing the number (or the total value) of items exchanged in the market.

- Minimizing the average waiting time of users in the system.

- Maximizing fairness in the system.

In order to achieve the above objectives we introduce virtual points and also a credit system. We prove that virtual points can decrease the average waiting time in the system and also assuming that users put their items a random permutation, there are instance in which in expectation the waiting time decreases by a factor of $2 k+2 B$.

Moreover, in order to achieve the fairness maximization goal, we define a credit system and we prove that not only is this problem NP-complete, but also not approximable within any multiplicative factor. Therefore, we propose two heuristics for this problem. The first one is an linear programmingrounding approach in which we use the results of the lp relaxation of the integer linear programming model of the problem. The other heuristic is a simple greedy algorithm.

For this chapter we perform experiments on the dataset that we had generated for Chapter 2's experiments. As mentioned above the dataset is generated according to power law distributions common in social networks. The experiments show that .... 


\subsection{Structure of the Thesis}

In this thesis our focus is on exchange markets which are an important example of online social networks. Chapters 2, and 3 study generation of recommendations for online exchange markets, in which the users have two sets of items. One set includes items which the user wishes for them (a.k.a wish-list) and the other is the list of items the user does not want anymore (a.k.a item-list). Chapter 2, models the network as a one-shot model and chapter 3 addresses the markets over time. 


\section{Chapter 2}

\section{One-shot Exchange Markets}

In this chapter we discuss One-shot exchange markets and focus on the problem of generating recommendations under two models: deterministic model and probabilistic model. In both models each user receives an item in return for the item which she gives away. We formulate the problem of finding recommendations as that of finding a set of conflict-free cycles in the graph. Conflict-free means cycles in the set are pairwise edge-disjoint, a condition necessary to ensure that when a user commits to a recommended exchange, she is not surprised that the recommended item is already taken. Thus, a recommendation consists of a set of conflict-free cycles. The value of a recommendation is the number of items (potentially) exchanged through the cycles recommended. This chapter is organized as follows. Related work are described in Section 2.1. The proposed exchange market models are described in Section 3.3, which also formalizes and illustrates the problems studied. The complexity of the problems is analyzed in Section 2.3. The heuristic and approximation algorithms are developed in Section 2.4 while their analysis is discussed in Section 2.5. Section 2.6 discusses the experiments. 


\subsection{Related Work}

The related work can be classified in the three following categories:

Cycle Cover Problem The cycle cover problem has been studied thoroughly. Given a graph and a subset of marked elements: nodes, edges or some combination of them, a cycle cover problem seeks to find a minimum length set of cycles whose union contains all marked elements [22]. In [22] the authors study cycle cover problem for cycles with bounded size which cover a subset of the edges of a graph. More specifically, they improve the trivial approximation factor of 2 to $1+\ln (2)$ in weighted graphs and $O(\operatorname{lnk})$ for uniform graphs. The Chinese postman problem was introduced by Guan [17] and Edmonds and Johnson [12] proposed a polynomial-time algorithm to solve the problem in undirected graphs. Papadimitriou [30] proved that the problem is NP-hard for mixed graphs. Later, Raghavachari and Veerasamy [33] gave a $\frac{3}{2}$-approximation for this instance of the problem. An NP-hard [39] variant of the Chinese postman problem, the minimum weight cycle cover problem, adds the constraint that covering cycles must be simple. The bounded cycle cover problem, which constrains cycles to be of bounded size as well as simple, was introduced by Hochbaum and Olinick [18]. They presented a heuristic for the problem along with empirical analysis. The lane covering problem was introduced by Ergun et al. [13], who gave a heuristic for the problem along with an empirical analysis. A variant on the cycle covering problem which imposes a lower bound on the 
size of each cycle has been studied as well [9]. Other covering problems include covering a graph by cliques [16]. The book by Zhang [41] reviews the literature.

Recommender Systems Extensive work has been done in the area of recommender systems in different research areas such as cognitive science [36], approximation theory [31], information retrieval [37] and also management science [28]. The field gained more importance in the mid 1990s with the emergence of collaborative filtering papers. [34, 35, 38].

Moreover, recommender systems are usually classified into the following categories, based on how recommendations are made [7]:

- Content-based recommendations: The user will be recommended items similar to the ones the user preferred in the past;

- Collaborative recommendations: The user will be recommended items that people with similar tastes and preferences liked in the past;

- Hybrid approaches: These methods combine collaborative and contentbased methods.

Combining trust-based and CF approaches is a direction of current research [7].

In addition to recommender systems that predict the absolute values of ratings that individual users would give to the yet unseen items (as discussed above), there has been work done on preference-based filtering, i.e., predicting the relative preferences of users $[11,14,25,26]$. Preference-based 
filtering techniques would focus on predicting the correct relative order of the items, rather than their individual ratings.

The Kidney Exchange Problem. A related problem in exchange markets is the national kidney exchange problem [5]. For many patients with kidney disease, the best option is to find a living donor, that is, a healthy person willing to donate one of his/her two kidneys. The problem is that frequently, a potential donor and his intended recipient are blood-type or tissue-type incompatible. In the past, the incompatible donor was sent home, leaving the patient to wait for a deceased-donor kidney. However, there are now a few regional kidney exchanges in the United States, in which patients can swap their willing but incompatible donors with each other, in order for each to obtain a compatible donor [5]. The kidney exchange problem is very similar to our problem, specifically the simple exchange market and the exchange market through short cycles. In kidney exchange, each patient needs one and only one kidney and each donor is willing to donate only one kidney(!). Because of medical constraints, one wants short exchange cycles. It is shown in [5] that (optimal) kidney exchange is NP-complete when exchange cycles of length $\leq k$ are considered, where $k>2$.

If exchanges are restricted to swaps, the kidney exchange problem can be solved using the maximum weighted perfect matching problem in which given an edge-weighted bipartite graph, we are looking for a maximum weighted perfect matching. As a result, the problem will be solved polynomially. Given a network $G=(V, E)$, a bipartite graph can be constructed as follows: put one vertex for each agent and one vertex for each correspond- 
ing item (i.e. kidney). Connect each agent to its corresponding item with an edge $e_{v}$ with weight zero. For each edge $e \in E, e=\left(v_{i}, v_{j}\right)$ in the original graph, connect agent $v_{i}$ to item $v_{j}$ with an edge with weight $w_{e}$. Perfect matchings in this model are equivalent to cycle covers, because receiving an item is equal to giving an item away, and finding a maximum weighted matching solves the problem of finding an optimal set of kidney swaps.

However, in all our exchange markets, users may have multiple items in their item and wish lists and may be willing to trade more than one item at a time. This subtle difference alone makes our problem much harder. As we show later, even finding optimal swap recommendations for simple market exchange is NP-complete.

\subsection{Model}

In our proposed system, we assume the algorithm for generating recommendations for exchange cycles is run periodically and the set of potential feasible exchanges are discovered. Users involved in these potential cycles are informed through email and/or their account will be updated. If they are interested in performing the transaction, they will get in touch with the other users. Otherwise they will withdraw the transaction and all other involved users will be informed. In any case when the transaction is done the exchanged items are removed from the item lists and wish lists, and the system will be updated. We also assume that a user does not own multiple copies of an item and also does not wish for multiple copies of an item.

Obviously the system is dynamic meaning that it changes with time. 
The possible changes are: (i) a user joins or leaves the network; (ii) an item is added to or removed from a user's wish list or item list: these changes may be due to a transaction done or due to change in user's interests.

These changes imply that the set of feasible exchanges will also change by time. Thus, the system runs the algorithm to find the set of new exchanges that have become available periodically (e.g., once a day or week). In this section, we formally define our problems. In the deterministic cases the objective is to maximize the number of items exchanged and in the probabilistic case to maximize the expected number of items exchanged. The following example illustrates many of the notions and will serve as a running example.

\section{Example 2.2.1 [Exchange Market]}

Consider a collection of five users - Alice, Bob, Joe, Amy, and Mary. Each of them has an item list and a wish list. The lists are shown in tables 2.1, $2.1,2.2,2.3,2.4$, and 2.5. Item lists of a user consists items the user is willing to give away in exchange for some item in their wish list that they would like to receive.

The tables indicating the users' wishlists and itemlists.

\begin{tabular}{|c|c|}
\hline Alice's Itemlist & Alice's Wishlist \\
\hline$B_{1}:$ Harry Potter I & $B_{2}:$ Harry Potter II \\
\hline Cook Book & $B_{3}:$ The secret \\
\hline & $B_{9}:$ PCs for dummies \\
\hline & $B_{8}:$ TAOCP I \\
\hline
\end{tabular}

Table 2.1: Alice's Item and Wish list 
Chapter 2. One-shot Exchange Markets

\begin{tabular}{|c|c|}
\hline Bob's Itemlist & Bob's Wishlist \\
\hline$B_{4}:$ CLRS & $B_{5}:$ Intro. to DBMS \\
\hline & $B_{7}:$ Cook Book \\
\hline
\end{tabular}

Table 2.2: Bob's Item and Wish list

\begin{tabular}{|c|c|}
\hline Joe's Itemlist & Joe's Wishlist \\
\hline$B_{2}:$ Harry Potter II & $B_{6}:$ Harry Potter III \\
\hline & $B_{3}:$ The secret \\
\hline
\end{tabular}

Table 2.3: Joe's Item and Wish list

In all our problems below, we assume a set of users $U$ and a set of items I. User $u \in U$ owns a set of items (aka item list) $S_{u}$, and requires a set of items (aka wish list) $W_{u}$.

Simple Exchange Market: In a simple exchange market, we only match up users one by one, i.e., in each exchange, two users $u$ and $v$ can exchange a pair of items $i$ and $j$. Given a set of users $U$ with the item lists $S_{u}$ and wish lists $W_{u}$ for each user $u \in U$, the simple market problem, denoted by SimpleMarket, is to find a set of pairs $[(u, i),(v, j)]$ where $i \in S_{u} \cap W_{v}, j \in S_{v} \cap W_{u}$. We call each pair a swap. The problem can be modeled as a directed graph $G=(V, E)$ with users as nodes $V$ and a directed edge $(u, v) \in E$ labeled $i$ whenever $i \in S_{u} \cap W_{v}$, i.e., $u$ owns item $i$ and $v$ is interested in receiving it. Thus, swaps correspond to cycles of length two. Since each user typically has one instance of an item in $S_{u}$ and also needs one instance from each item in $W_{u},[(u, i),(*, *)]$ should appear in the set at most once, where the first $*$ is any other user $v \neq u$ and the second $*$ is any item. In terms of graphs, the configuration shown in Figure 2.1 is forbidden, i.e., the set of recommended swaps should not contain this as a subgraph. We call a recommendation, i.e., set of swaps, without 
Chapter 2. One-shot Exchange Markets

\begin{tabular}{|c|c|}
\hline Amy's Itemlist & Amy's Wishlist \\
\hline$B_{3}:$ The Secret & $B_{2}:$ Harry Potter II \\
\hline$B_{8}:$ TAOCP I & $B_{4}:$ CLRS \\
\hline
\end{tabular}

Table 2.4: Amy's Item and Wish list

\begin{tabular}{|c|c|}
\hline Mary's Itemlist & Mary's Wishlist \\
\hline$B_{9}:$ PCs for Dummies & $B_{2}:$ TAOCP I \\
\hline & $B_{10}:$ TAOCP II \\
\hline
\end{tabular}

Table 2.5: Mary's Item and Wish list

such a forbidden configuration conflict-free. To see why conflict-freedom is necessary, consider recommending both pairs $[(u, i),(v, j)]$ and $[(u, i),(w, j)]$ to the users. If user $u$ exchanges $i$ for $j$ with user $v$, then the recommended exchange is no longer feasible for user $w$. Thus, set of recommended exchanges contains a conflict. The condition above ensures it is conflict-free. Conflict-free recommendations ensure that users do not get turned off or lose their trust in their system by finding out that a recommendation they received from the system is no longer feasible. In our running example (Example 2.2.1), Joe can give away $B_{2}$ to both Amy and Alice, but both edges cannot be in the graph at the same time.

Exchange Markets through Short Cycles. In an online exchange market, we can find cycles of size larger than 2, for example, as can be seen in Figure 2.2, we may find users Alice, Bob and Amy with items $B_{7}, B_{4}$, and $B_{8}$. If Alice, Bob and Amy have items $B_{8}, B_{7}$, and $B_{4}$ respectively in their wish lists, we can set up a 3-way exchange among them and have all of them satisfy their wish lists. Note that, in this example, if we restrict ourselves to swaps, none of the users may be satisfied. An exchange cycle is a sequence 


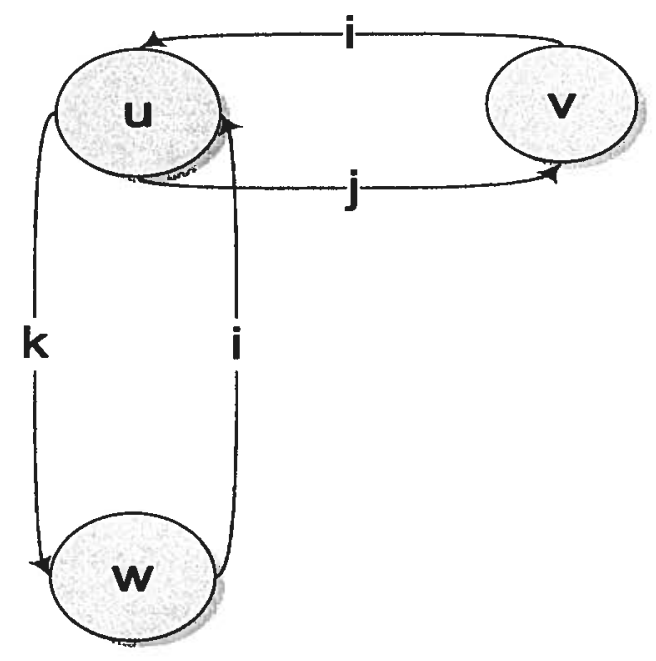

Figure 2.1: Both exchanges cannot be present in the system at the same time.

$\left[\left(u_{1}, i_{1}\right),\left(u_{2}, i_{2}\right), \ldots,\left(u_{k}, i_{k}\right)\right]$, with $i_{j} \in S_{u_{j}} \cap W_{u_{j \oplus 1}}$, where $j \oplus 1=j+1$, $1 \leq j<k$, and $k \oplus 1=1$. A set of exchange cycles is said to be conflict-free provided the pattern $[(u, i),(*, *)]$ appears at most once in the set, i.e., the corresponding graph does not contain the forbidden subgraph in Figure 2.1.

Our goal is to find an optimal set of conflict-free cycles: The objective is to maximize the number of items involved in exchanges, thus maximizing the number of transactions. Furthermore, in practice, we may wish to limit the length of the exchange cycles to a maximum of $k$, where $k$ is some predetermined constant. We usually consider $k=2, \ldots, 5$. The reason why we focus on short cycles is that, even if one user in the cycle withdraws the transaction, the whole exchange cycle collapses. If we have long cycles, then more users would be affected. 


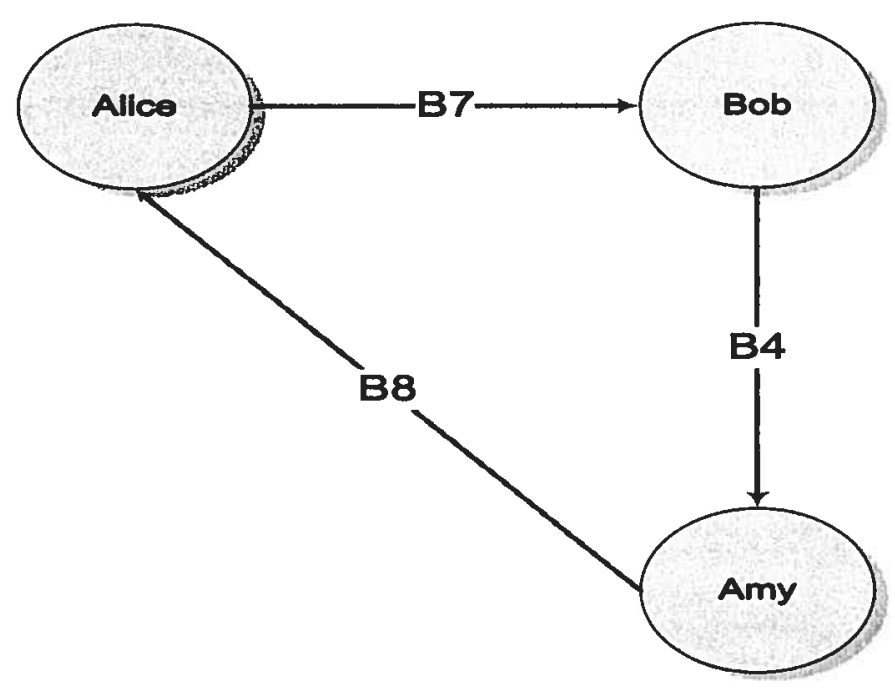

Figure 2.2: Exchange cycle of length three: [(Alice, $\left.B_{7}\right)$, (Bob, $\left.B_{4}\right)$, (Amy, $\left.B_{8}\right)$ ]

In our running example, we can see that Alice has $B_{7}$ in her item list and Bob has it in his wish list. Bob has $B_{4}$ in his item list and Amy has wished for that book. At the same time $B_{8}$ in Amy's item list while Alice is interested in $B_{8}$. As we see the cycle [(Alice, $\left.B_{7}\right)$, (Bob, $\left.B_{4}\right)$, (Amy, $\left.B_{8}\right)$ ] is a feasible cycle.

Probabilistic exchange markets. Formalizing the above problem in a probabilistic setting, we can assume that using a reputation system, we can estimate the probability of realizing each exchange in the exchange market graph. Reputation systems have become one of the essential components of 
web-based multi-agent systems. These systems gate agents reviews of one another, as well as about external events, into valuable information [32]. As a result, we have a graph with some probability on each edge. Assuming that the probability of realizing each edge is independent of other edges, our goal is to find a set of cycles with the maximum expected number of edges covered, since each edge corresponds to an item being exchanged. Note that the probability of a cycle is the product of the probability of its edges. It would be interesting to understand the complexity of this problem. The probability $P_{u}(v)$ denotes the probability that $u$ is willing to do an exchange with user $v$, and $P^{u}(i, j)$ is the probability that user $u$ will exchange item $i$ with item $j$, i.e., she gives $i$ and takes $j$. In this case, the probability of a cycle being realized will be

$$
\begin{aligned}
& P_{u_{1}}\left(u_{2}\right) \times P^{u_{1}}\left(i_{1}, i_{k}\right) \times P_{u_{2}}\left(u_{3}\right) \times P^{u_{2}}\left(i_{2}, i_{1}\right) \ldots \times \\
& P_{u_{k}}\left(u_{1}\right) \times P^{u_{k}}\left(i_{k}, i_{k-1}\right) .
\end{aligned}
$$

Our goal is to find cycles that maximize the total expected number of items exchanged.

\section{Example 2.2.2 [Probabilistic Exchange Market]}

Consider Figure 2.2 as an example. Let the probabilities associated with the edges (Alice, Bob), (Bob, Amy) and (Amy, Alice) be 0.7, 0.55 and 0.9. For simplicity, suppose the probability of this cycle being realized will be $0.7 \times 0.55 \times 0.9=0.3465$. Let $R$ be a recommendation including this exchange cycle among others. Then the probability of this cycle being realized will be $0.7 \times 0.55 \times 0.9=0.3465$. Let $R$ be a recommendation including this exchange cycle among others. Then the contribution of this cycle to the value of $R$, 
i.e., to the expected number of exchanged items is $3 \times 0.3465=1.0395$.

In almost all of the cases, the probability of each edge being realized is less than one, so when the cycle gets larger, the probability would be very low. Therefore (similar to what we argued before for the deterministic case) in the probabilistic case short cycles are practically more appealing.

\subsection{Hardness Results}

In this section, we show that the SimpleMarket problem is NP-complete. We also show that the ProbMarket problem is NP-complete, regardless of whether we consider swaps or short cycles with length bounded by a constant $k>2$. Even the "kidney exchange version" of ProbMarket where every user owns only one item and wishes for only one item remains NP-complete when cycles of length $>2$ are allowed. We first define the decision versions of these problems formally.

\begin{tabular}{|l|} 
SimpleMarket Decision Problem \\
Instance: A set of users $U$ with the item lists \\
$S_{u}$ and wish lists $W_{u}$ for each user $u \in U$. \\
Question: Does $\exists$ a conflict-free swap cover \\
$C$ with number of items exchanged $\geq K ?$
\end{tabular}

The simple market decision version, Probusr associates with every user a probability that she will transact with any user. Prob ${ }_{i t m}$ associates with each user and a pair of items $i, j$, a probability that the user will give $i$ and take $j$ in exchange. 
Chapter 2. One-shot Exchange Markets

ProbMarket Problem
Instance: A set of users $U$ with the item
lists $S_{u}$ and wish lists $W_{u}$ for each user $u \in$
$U$ and two probability assignment functions
Prob $_{u s r}: U \times U \rightarrow[0,1]$ and Prob $_{i \text { tm }}: U \times$
$I \times I \rightarrow[0,1]$.
Question: Does $\exists$ a cycle cover $C$ whose ex-
pected number of items is $\geq K ?$

We next define TriEdgePart, a well-known NP-complete problem. We will reduce this problem to our problem.

TriEdgePart Problem
Instance: A tripartite graph $G$ with three
vertex partitions $(X, Y, Z)$ and an edge set
$E \subset X \times Y \cup X \times Z \cup Y \times Z$
Question: Does $\exists$ an edge partitioning of $G$
into disjoint sets of cycles of size three?

Finally, we define $4 C y c E d g e P a r t$, a problem involving 4-partite graphs. We will find it convenient to use this problem as an intermediary in our reductions. 
Chapter 2. One-shot Exchange Markets

4CycEdgePart Problem
Instance: A 4-partite graph $G$ with four ver-
tex partitions $(X, Y, I, J)$ and a collection of
edges $E$ consisting of a subset of edges from
$X \times Y \cup X \times I \cup Y \times J$, together with a multiset
of edges from $I \times J$.
Question: Does $\exists$ an edge partitioning of $G$
into disjoint sets of cycles of size four?

We first show that the $4 C y c E d g e P a r t$ problem is NP-complete and then give a reduction from $4 C y c E d g e P a r t$ to SimpleMarket showing NPcompleteness of SimpleMarket.

Theorem 1 The $4 C y c E d g e P a r t$ problem is NP-complete.

Proof: The membership in NP is straightforward: given an edge partitioning $P$ of $G$, we can check in polynomial time whether $P$ consists of 4-cycles that are pairwise edge-disjoint, covering all edges of $G$. To prove the hardness, we give a reduction from TriEdgePart. The triangle edgepartitioning of tripartite graphs is known to be NP-complete (and in fact APX-hard) by a result of Abraham et. al [6], following an NP-completeness by Holyer [20].

Given an instance $G(X \cup Y \cup Z, E)$ of TriEdgePart, we construct an instance $G^{\prime}\left(X^{\prime} \cup Y^{\prime} \cup I^{\prime} \cup J^{\prime}, E^{\prime}\right)$ of $4 C y c E d g e P a r t$ as follows: for any vertex $w \in Z$, we add two corresponding vertices $w_{1} \in I^{\prime}$ and $w_{2} \in J^{\prime}$; for each $u \in X$, we add a vertex $u^{\prime} \in X^{\prime}$; and for any $v \in Y$, we add a vertex $v^{\prime} \in Y^{\prime}$. 
In other words, we let

$$
\left|X^{\prime}\right|=|X|,\left|Y^{\prime}\right|=|Y|,\left|I^{\prime}\right|=\left|J^{\prime}\right|=|Z|
$$

, i.e., we choose $X^{\prime}, Y^{\prime}$ to be any sets with size $|X|,|Y|$, and $I^{\prime}$ and $J^{\prime}$ to be any sets with size $|Z|$, with $X^{\prime}, Y^{\prime}, I^{\prime}, J^{\prime}$ being pairwise disjoint. Then, we construct edges $E^{\prime}\left(G^{\prime}\right)$ of the new instance as follows: for any edge $(u, v) \in E(G)$ where $u \in X$ and $v \in Y$, we add an edge $\left(u^{\prime}, v^{\prime}\right)$ to $E^{\prime}\left(G^{\prime}\right)$; for any edge $(u, w) \in E(G)$ where $u \in X$ and $w \in Z$, we add an edge $\left(u^{\prime}, w_{1}\right)$ to $E^{\prime}\left(G^{\prime}\right)$; and for any edge $(v, w) \in E(G)$ where $v \in Y$ and $w \in Z$, we add an edge $\left(v^{\prime}, w_{2}\right)$ to $E^{\prime}\left(G^{\prime}\right)$. Finally, we add $\frac{\operatorname{deg}(w)}{2}$ edges between every pair $w_{1} \in I^{\prime}$ and $w_{2} \in J^{\prime}$ (corresponding to a vertex $w \in Z$ ). Note that $\operatorname{deg}(w)$ is even in our case, otherwise it is clear that there is no triangle partitioning. The reason that we put $\frac{\operatorname{deg}(w)}{2}$ edges is that there may be more than one triangle passing through $w \in Z$, and each triangle corresponds to two edges for passing through any node, therefore to maintain the same number of cycles in the 4-partite graph, we need to connect $w_{1}$ and $w_{2}$ with $\frac{\operatorname{deg}(w)}{2}$ edges. Clearly, $G\left(X^{\prime} \cup Y^{\prime} \cup I^{\prime} \cup J^{\prime}, E^{\prime}\right)$ is an instance of the $4 C y c E d g e$ Part problem. It should also be noted that while instances of the TriEdgePart are always simple graphs, instances of the 4 CycEdgePart can be multi-graphs since we need multiple edges between $w_{1}$ and $w_{2}$, for various $w \in Z$. However,they are not arbitrary multi-graphs: e.g., between $x \in X$ and $y \in Y$ at most one edge is required (see the formal definition of the 4 CycEdgePart decision problem above). 
Now, we formally show that there exists an edge-partitioning of edges of $G$ into edge-disjoint triangles if and only if there exists an edge-partitioning of $G^{\prime}$ into edge-disjoint 4-cycles. Consider an edge-partitioning $T$ of edges of $G$ to edge-disjoint triangles $T=\left\{\left(\left(x_{1} y_{1} z_{1}\right), \ldots,\left(x_{p} y_{p} z_{p}\right)\right)\right\}$ for $p=\frac{\lfloor E(G)\rfloor}{3}$. For each triangle $x_{i} y_{i} z_{i}$ in this edge-partitioning $T$ where $x_{i} \in, y_{i} \in Y$, and $z_{i} \in Z$, we associate a corresponding 4-cycle $x^{\prime} y^{\prime} z_{1} z_{2}$ in $G^{\prime}$ where $x^{\prime} \in X^{\prime}$, $y^{\prime} \in Y^{\prime}$, and $z_{1} \in I^{\prime}$, and $z_{2} \in J^{\prime}$. Since the edges of $p$ triangle $\left(x_{i} y_{i} z_{i}\right)$ for $1 \leq i \leq p$ cover all edges of $G$, each vertex $z \in Z$ appears exactly in $\frac{\operatorname{deg}(z)}{2}$ triangles. Let $T^{\prime}$ be the set of corresponding 4-cycles to triangles in $T$. Thus, there are exactly $\frac{\operatorname{deg}(z)}{2} 4$-cycles in $T^{\prime}$ corresponding to triangles in $T$, and as a result, $T^{\prime}$ is also an edge-partitioning of $G^{\prime}$ into edge-disjoint 4-cycles. Now, consider an edge-partitioning $T^{\prime}$ of $G^{\prime}$ into edge-disjoint triangles. Similar to the above construction, we can construct a set $T$ of triangles partitioning the edges of $G$ to $p$ edge-disjoint triangles. The above two facts show that there exists an edge-partitioning of edges of $G$ to edge-disjoint triangles if and only if there exists an edge-partitioning of $G^{\prime}$ to edge-disjoint 4-cycles. This completes the proof of hardness.

We next show:

Theorem 2 The SimpleMarket problem is NP-complete.

Proof: Once again, membership in NP is straightforward: given a set swaps, it is easy to check whether it is conflict-free and the number of items covered is $\geq K$. To prove the hardness, we give a reduction from 4 CycEdgePart to the SimpleMarket problem. 


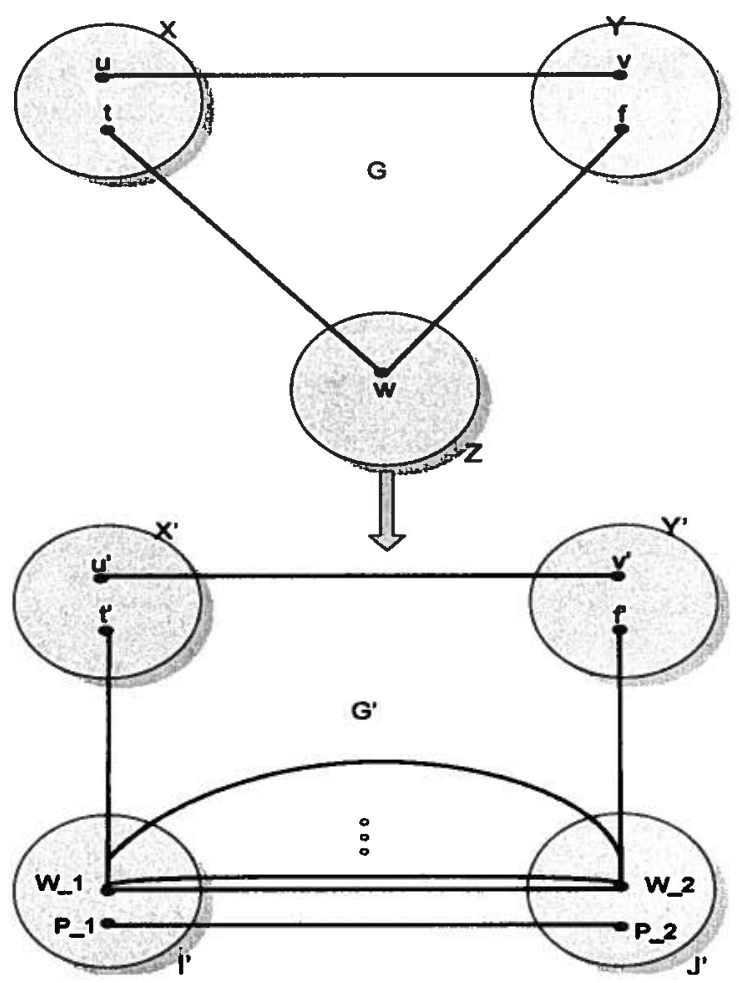

Figure 2.3: Hardness Proof 
Given an instance $G(X \cup Y \cup I \cup J, E)$ of the $4 C y c E d g e P a r t$ problem, we construct an instance $\Sigma$ of the simple marketing problem as follows: we let the set of users be $X \cup J$, and the set of items be $I \cup Y$. For each user $x \in X$, we set the item list of $x$ to $S_{x}=\{i \in I \mid(x, i) \in E(G)\}$ and the wish list of $x$ to $W_{x}=\{y \in J \mid(x, y) \in E(G)\}$. Similarly, for each user $j \in J$, we set the item list of $j$ to $S_{j}=\{y \in Y \mid(y, j) \in E(G)\}$ and the wish list of $j$ to $W_{j}=\{i \in I \mid(i, j) \in E(G)\}$.

Now, we show that there exists an edge-partitioning of $G$ into $p=\frac{E(G)}{4}$ edge-disjoint 4-cycles if and only if there exists a conflict-free set of $p$ swaps between pairs of users in the instance $\Sigma$ of the simple market exchange problem. Consider an edge-partitioning $T$ of $G$ into $p$ edge-disjoint 4-cycles $T=\left\{\left(x_{v}, y_{v}, j_{v}, i_{v}\right) \mid 1 \leq v \leq p\right\}$. In any 4-cycle $\left(x_{v}, y_{v}, i_{v}, j_{v}\right)$ clearly $x_{v} \in X$, $y_{v} \in Y, i_{v} \in I$, and $j_{v} \in J$. Consider the corresponding possible swap where $x_{v}$ gives item $i_{v}$ to user $j_{v}$ and receives item $y_{v}$ in return from $j_{v}$. By the construction of item and wish lists, this swap is feasible. Let $\mathcal{S}$ be the set of swaps derived from $T$. Clearly $|\mathcal{S}|=p$. We show $\mathcal{S}$ is conflict-free. Suppose not. Then there is a pair of swaps in $\mathcal{S}$ of the form: user $a$ swaps item $\alpha$ for item $\beta$ with user $b$ and $a$ also trades $\alpha$ for item $\gamma$ with user $c$ (which may or may not be $b$ ). This implies $T$ contains two 4-cycles which share the edge $(a, \alpha)$, a contradiction to the edge-disjointness of $T$.

Now, suppose $\mathcal{S}$ is any conflict-free set of $p$ swaps for the instance $\Sigma$. We construct an edge-disjoint 4-cycle cover $T$ as follows. For every swap where user $a$ trades $\alpha$ for $\beta$ with user $b$, choose the corresponding 4-cycle $(a, \alpha, b, \beta, a)$. It is easy to show $T$ covers all edges of $G$ and it is pairwise edge-disjoint. This completes the reduction and the proof. $\mathbf{}$ 
Chapter 2. One-shot Exchange Markets

We next turn our attention to the ProbMarket problem. The following restricted versions are particularly interesting. First, what can we say about ProbMarket when we restrict attention to swaps, i.e., when we are interested in finding an optimal conflict-free set of swaps? The hardness for the corresponding deterministic case implies the probabilistic version is hard as well. In particular, the deterministic version is a special case when all probabilities are set to 1 . Second, suppose every user owns just one item and wishes for just one item. This is similar to the kidney exchange problem and we call it the "kidney exchange version" of ProbMarket. If exchange cycles with length more than two are allowed, by the result of [5], it follows that this problem is NP-complete. When only swaps are allowed, the same technique of finding maximum weighted perfect matching can be used to solve this problem exactly in polynomial time. We thus have:

Corollary 1 Finding an optimal set of swaps for ProbMarket is in general NP-complete. However, for the kidney exchange version, the problem can be solved in polynomial time. Finding an optimal conflict-free set of exchange cycles where cycle length is bounded by $k>2$ for the kidney exchange version of ProbMarket is NP-complete.

\subsection{The Algorithms}

In this section, we develop four algorithms for variants of the exchange market problem. Three of them have provable approximation bounds w.r.t. optimal algorithm. The remaining one is a heuristic algorithm with no such guarantees. However, as we will see shortly, it is much more efficient than the 
approximation algorithms. While theoretically the heuristic algorithm may not sound interesting compared to the approximation ones, we study their relative performance on synthetically generated data sets in Section 2.6.

Some of these algorithms are inspired by algorithms for the set packing problem. In section 2.5 , we define the weighted $\mathrm{k}$-set packing problem and show that the exchange market problem can be seen as a special case of the set packing problem. We exploit this connection to derive the approximation bounds.

In the rest of the paper, we will find it convenient to deal with a graph representation of market exchange problems. Given a (simple or shortcycles) market exchange problem instance $\Sigma=\left(U, I,\left\{S_{u} \mid u \in U\right\},\left\{W_{u} \mid\right.\right.$ $u \in U\}$ ), with users $U$, items $I$, item lists $S_{u}$ and wish lists $W_{u}$, the corresponding graph representation $G_{\Sigma}$ is defined as follows. $G_{\Sigma}$ is a directed edge-labeled graph and has a node for every user in $U$. There is a directed edge $(u, v)$ labeled $i$ whenever $i \in I$ is an item such that $i \in S_{u} \cap W_{v}$. For our running example of Example 2.2.1, the corresponding graph is shown in Figure 2.4.

\subsubsection{Algorithm Maximal}

One heuristic algorithm is to look for a maximal set of cycles in $G_{\Sigma} . S$ is a maximal set of cycles if $S$ is conflict-free and there exists no cycle $C$ in $G_{\Sigma}$ which does not appear in $S$ and does not conflict with any cycle in $S$.

\section{Example 2.4.1 [Maximal vs. Optimal]}

Let's consider the following cycles in the running example: 


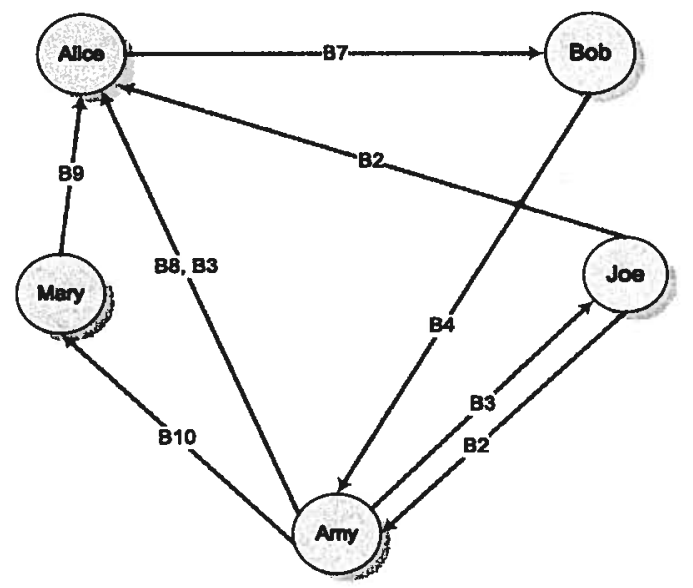

Figure 2.4: Graph Representation of Running Example. 
$C_{1}=\left[\left(\right.\right.$ Alice,$\left.B_{7}\right),\left(\mathrm{Bob}, B_{4}\right),\left(\right.$ Amy, $\left.\left.B_{8}\right)\right], C_{2}=\left[\left(\right.\right.$ Alice,$\left.B_{7}\right),\left(\right.$ Bob,$\left.B_{4}\right)$, $\left(\right.$ Amy, $\left.B_{10}\right)$, (Mary, $\left.\left.B_{9}\right)\right]$, and $C_{3}=\left[\left(\right.\right.$ Amy, $\left.B_{3}\right),\left(\right.$ Joe, $\left.\left.B_{2}\right)\right]$

Two sets $S_{1}$ and $S_{2}$ are maximal:

$S_{1}=\left\{c_{1}, c_{3}\right\}$ and $S_{2}=\left\{c_{2}, c_{3}\right\}$. Note: coverage $\left(S_{1}\right)=\left\{B_{7}, B_{4}, B_{8}, B_{2}, B_{3}\right\}$ and coverage $\left(S_{2}\right)=\left\{B_{7}, B_{4}, B_{10}, B_{9}, B_{2}, B_{3}\right\}$. Both are maximal since no cycles can be added to either without breaking the conflict-freedom property. Furthermore, notice that coverage $\left(S_{1}\right) \nsubseteq \operatorname{coverage}\left(S_{2}\right)$ and coverage $\left(S_{2}\right) \nsubseteq$ coverage $\left(S_{1}\right)$. However, $S_{1}$ is clearly not optimal since $S_{2}$ covers strictly more items.

In order to find the maximal set of cycles, we first initialize the set of cycles $\mathcal{B}$ to $\emptyset$. Then for a random node $v \in V(G)$ we perform a breadth first search (BFS) or a depth first search (DFS) from $v$ to find a cycle. While performing the BFS/DFS the first time that we encounter a backward edge we can stop because a cycle has been found. BFS will find the shortest cycle. And terminate if no cycles are found. Add $C$ to $\mathcal{B}$, and remove all edges that are in conflict with $C$ from the graph. Set $C$ to $\emptyset$ again and start the BFS from another node. We repeat this procedure $M$ times and select the result with maximum weight. In Section 2.6, we explore the performance of this algorithm for different choices of $M$. The complete description of Algorithm Maximal is depicted in Figure 2.4.1.

\subsubsection{Greedy Algorithm}

Another approach is to perform a greedy algorithm. Initialize $\mathcal{B}$ to $\emptyset$. At each step find the best exchange cycle $C$ with the maximum weight. In 
order to find the best cycle, we should try all short cycles and then pick the cycle with maximum weight. Then add $C$ to the set of cycles $\mathcal{B}$. Remove all the edges that are in conflict with $C$. Add $C$ to $\mathcal{B}$ and if no cycles are found terminate. The complete description of Algorithm Greedy is depicted in Figure 2.4.2.

\subsubsection{Local Search Algorithm}

In this algorithm we attempt to replace a small subset of the current solution by some set of cycles that result in a greater total weight. Let the current solution be $\mathcal{B}$. For any exchange cycle $C$ that is not already selected, try to add $C$ and remove all the conflicting edges. If the total weight of $\mathcal{B}$ increases by a factor $\beta$, add $C$ to $\mathcal{B}$ and update $\mathcal{B}$ by removing all conflicting cycles from $\mathcal{B}$. Do this procedure until no local improvement is possible, output $\mathcal{B}$ and terminate. The complete description of Algorithm Local Search is depicted in Figure 2.4.3.

\subsubsection{Greedy/Local Search}

Instead of starting the Local Search algorithm with an empty set, we can seed it with a good set of cycles. A variant is to run the greedy algorithm to find a set of cycles $\mathcal{B}$ and then run the local search algorithm starting from cycles in $\mathcal{B}$. Analysis of this variant is presented in Section 2.5. 


\subsection{Analysis}

\subsubsection{Performance}

In order to prove approximation factors for the algorithms proposed in this paper we link our problems to the weighted $k$-set packing problem. We show that the simple market exchange problem can be formalized as a special case of the weighted $k$-set packing problem. Note that the purpose of this formulation is solely for the purpose of deriving approximation bounds.

In the weighted $k$-set packing problem, given a collection of sets, each of which has an associated weight and contains at most $k$ elements drawn from a finite base set, our goal is to find a collection of disjoint sets of maximum total weight. The restriction to sets of size at most $k$ properly includes multi-dimensional matching, which is a generalization of the ordinary graph matching problem. Weighted $k$-set packing is proved to be NP-hard for any $k \geq 3$, even in the unweighted case [15], and heuristics and approximation algorithms have been developed for this problem.

To formulate the one-shot exchange market problem as a special case of the set packing problem, we define the elements of the sets to consist of the user, the item, and the act of giving or wishing. The formulation is as follows:

First, enumerate all exchange cycles of length $\leq k$. This can be done in polynomial time since $k$ is a constant. Construct a set corresponding to every exchange cycle. To illustrate, consider an exchange cycle where user $u$ gives item $i$ to user $v$ and wishes item $j$ in return. The elements of the set are: 
( $u$ gives $i$ ) denoted by $\mathbf{x}_{u, i}$.

$\left(v\right.$ gives $j$ ) be denoted by $\mathbf{x}_{v, j}$.

( $u$ wishes $j$ ) be denoted by $\mathbf{y}_{u, j}$.

( $v$ wishes $i$ ) be denoted by $\mathbf{y}_{v, i}$.

Thus, the set corresponding to the above exchange is

$$
\left\{\mathbf{x}_{u, i}, \mathbf{x}_{v, j}, \mathbf{y}_{u, j}, \mathbf{y}_{v, i}\right\}
$$

In the above notation, $\mathbf{x}$ is a symbol for "giving an item" and $\mathbf{y}$ for "wishing an item". The first term in the subscript shows the user involved in gives/wishes relation and the second term is the item being exchanged.

Set the weight of each set constructed to be the cardinality of the set. For a probabilistic variant, set the weight to be the cardinality times the product of probabilities associated with the exchange cycle. Let $w(C)$ denote the weight of a cycle (equivalently, set) $C$.

In each of the exchange problems, our objective is to find a conflict-free set of exchange cycles $\mathcal{B}$ such that $\Sigma_{C \in \mathcal{B}} w(C)$ is maximized. This exactly corresponds to weighted $k$-set packing.

We proved in Section 2.3 that the simplest case of our problem which is the simple marketing problem is NP-complete. We also showed that the probabilistic exchange market problem is NP-complete. Here, using the above reduction to the $k$-set packing problem, we obtain approximation bounds on Algorithms Greedy, Local Search and Greedy/Local Search. The approximation bounds proved in the literature for these algorithms for 
weighted $k$-set packing carry over to our exchange problems since the latter can be seen as a special case of weighted $k$-set packing.

- Algorithm Maximal: It is not obvious how close the solution of this algorithm will be to the optimal solution. The reason is that the BFS algorithm is performed from an arbitrary node and picks the first cycle that is found.

- Algorithm Greedy: In [10], the authors show that performance of a greedy approach to the weighted $k$-set packing problem gives us a $2 k$ approximation. That is, in the worst case total weight of the output would be $\frac{1}{2 k}$ of the total weight of the set of maximum weights. The factor $2 k$ is because from the sets that are removed in each iteration, the optimal solution can contain at most $2 k$ sets, at most one for each element of the selected set all of which are of weight at most that of our selected set. They also state that this factor cannot be improved.

- Algorithm Local Search : Using a result in [8] we can show that this algorithm is a $2 k-1$-approximation, meaning that in the worst case the total weight of the given solution would be $1 /(2 k-1)$ of the total weight of the optimal solution.

- Local Search/Greedy Algorithm: In [10] the local search/greedy algorithm is proved to have a performance ratio of $2(2 k+1) / 3$ and that this ratio is asymptotically tight. 


\subsubsection{Running Time}

In the following, the number of cycles that are found is depicted by $|\mathcal{B}|$. The worst case running time of the proposed algorithms are as follows:

- Maximal Algorithm: The running time of this algorithm is $O((|V|+$ $|E|)|\mathcal{B}|)$. Each BFS will take $O(|V|+|E|)$ time. In the worst case the number of cycles that are found is $|\mathcal{B}|$.

- Greedy Algorithm: Finding the best cycle in each step takes $O\left(|V|^{2 k}\right)$ time, thus the total running time will be $O\left(|V|^{2 k}|\mathcal{B}|\right)$

- Local Search: In the local search algorithm, the running time of checking all cycles is $O\left(|V|^{2 k}\right)$. In order to check if each cycle is in conflict with the cycles in the current solution at most $O(|E|)$ time is needed. At each step, we perform the local operation if it increases the weight of the solution by more than a $1+\epsilon$ factor, where $\epsilon$ is a small constant. Therefore, the number of local operations will be $\log _{1+\epsilon}$ OPT where OPT is the weight of the optimum solution. Thus the worst case running time for the local search algorithm will be: $O\left(|V|^{2 k}|E| \log\right.$ OPT).

- Greedy/Local Search: The running time of this algorithm simply is the addition of the greedy and local search algorithm, because the results of the greedy algorithm will be input to the local search algorithm.

\subsection{Experimental Results}

We implemented the Maximal, Greedy, and Local Search algorithms using MATLAB. The experiments were run on a Computer with a $2.16 \mathrm{GHz}$ Intel 
Core 2 Duo CPU and 1 GB of RAM under Windows XP.

The goals of our experiments are as follows:

- Obviously, allowing exchange cycles of length more than two will make for increased coverage of users/items. We wish to study the impact of allowing cycles of length more than two on the extent to which coverage increases.

- To examine the quality of the results found by algorithms, especially the Maximal algorithm which does not have a guaranteed approximation factor.

- To study the scalability of Algorithm Maximal and also the impact of the parameter $M$ (number of repeated trials) on the quality of the output.

\subsubsection{Data Set}

We could not get access to the data of real online exchange applications. Hence, we generated a set of synthetic data. The intuition behind the data generation is that the popularity of items in wish lists and item lists follow some power law distributions, i.e., there are many items which are wished for or provided by a small number of people, and there is a small number of items which are provided or wished for by many people. To achieve this goal, first, we generate some power law distributions with a given power as the parameter. We actually examined four different powers of $0.5,1,1.5$ and 2. We use one of these power law distributions for the popularity of the items, i.e., the number of people who own one item. We also generate a 
set of item list sizes based on some other power law vectors (this is justified by the fact that the size of wish lists and item lists should also follow some power law distribution). We also generate a vector of wish list size in direct correlation with the vector of item list sizes. The intuition here is that if a user provides more items, then probably she has larger wish lists as well. This intuition is not true in all cases, so we add some noise to this process with a small probability.

Now, given a popularity vector, and the two vectors of the wish list and item list sizes, we generate the real wish lists and item lists as follows. For each item to be added to an item list or wish list, we generate an item independently from the popularity vector. This process ensures that the expected number of appearances of items in item lists and wish lists follow some power law distribution.

\subsubsection{Experiments}

In order to achieve our experimental goals above, we performed the following experiment. First we just considered cycles of length two $(l=2)$ and observed the number of items involved in cycles. In the next steps we allow cycles of length three $(l=3)$, four $(l=4)$ and then five $(l=5)$ and measured the increase in the number of items covered compared to the previous cases. We didn't go beyond $l=5$ since as mentioned in Section 3.3 long cycles are not of interest in the market exchange problems studied in this paper. We ran our algorithms on three different data sets with 10,000, 25,000 and 50,000 users.

Table 2.6 summarizes the results of the algorithms in form of average 
amount of increase in coverage as the allowed cycle length is increased from 2 to 5 . It is interesting to note that the extent of incremental coverage drops as the allowed cycle length $l$ is increased from 2 to 5 . This is consistent for all three algorithms. This can be explained by the small world phenomenon of networks following power law distributions. Beyond a certain length, cycles of longer length tend to pay "diminishing returns" in terms of \%increase in the coverage. The figures in the table correspond to the data set of size 10,000 , averaged over various $\alpha=0.5,1.0,1.5,2.0$. We observed little variance over different values of $\alpha$.

\begin{tabular}{|c|c|c|c|}
\hline \% Increase & $2 \rightarrow 3$ & $3 \rightarrow 4$ & $4 \rightarrow 5$ \\
\hline Maximal Alg. & 5.56 & 3.40 & 2.7 \\
\hline Greedy Alg. & 7.33 & 3.81 & 3.12 \\
\hline Local Search Alg. & 7.71 & 3.85 & 3.35 \\
\hline
\end{tabular}

Table 2.6: \% Increase in coverage when considering cycles of length 3,4 and 5.

Figures 2.8, 2.9 and 2.10 illustrate results of the experiments for maximal, greedy and local search algorithms respectively. In all figures, "size of data set" refers to the number of users.

First, for any of the algorithms, the number of items covered for any fixed value of $l$ decreases as the skew factor $\alpha$ increases. E.g., in Figure 2.8, at $\alpha=0.5$ and $l=4$, about 50,000 items are covered by the recommendations generated by Algorithm Maximal on the data set of size 25,000. On the same data set and $l$, at $\alpha=2.0$, this number drops to about 15,000 . The same trend can be observed in the plots for other algorithms. The reason this happens is as the data is more skewed, fewer users own or wish for any reasonable number of items. Thus, the number of users who can engage in 
Chapter 2. One-shot Exchange Markets

fruitful exchanges decreases which in turn brings down the number of items covered.

Second, while theoretically, Algorithm Maximal does not enjoy any provable approximation guarantees, the quality of its output is comparable to that of the other algorithms. E.g., consider Figures 2.8, 2.9, and 2.10 and examine the number of items covered by the algorithms for the following situation: $l=4$ and $\alpha=1,1.5$, and data set size $=25,000$. For $\alpha=1.0$, the maximal algorithm achieves a coverage of about 60,000 items whereas both greedy and local search achieve about 65,000. Similarly, for $\alpha=1.5$, maximal achieves about 35,000 compared to about 41,000 achieved by both greedy and local search.

Next, Figure 2.12 compares the average of the running times of the algorithms. The figure shows the running times averaged over various values of $\alpha$. As expected, the running time of the Maximal algorithm is much better than that of the two other algorithms. Taken together with the fact that the quality of the output of Algorithm Maximal is found to be comparable to that of the other algorithms, this makes it a serious contender for finding a conflict-free set of exchange cycles with good coverage.

To compare running time of the algorithms according to different values of $\alpha$, we plot the running times of the algorithms on datasets of size 10000 , 25,000 and 50,000 over various values of $\alpha=0.5,1,1.5$, and 2. As we can see in all Figures $2.13,2.14,2.15,2.16,2.17,2.18,2.19,2.20$, and 2.21 running time of the algorithms decreases as the value of $\alpha$ increases.

Another parameter that was explored was the number of times the maximal algorithm (denoted as $M$ in the Section 2.4) is run in order to 
Chapter 2. One-shot Exchange Markets

get a reasonable result quality in a reasonable time. We examined $M=$ $20,50,100,120$. The results are illustrated in Figure 2.11. The result quality, measured in number of items covered, improves significantly when $M$ is increased from 20 to 50 . From 50 to 100 , the increase drops and finally from 100 to 120 , the increase is almost negligible. We selected $M=100$ in all our experiments.

\subsection{Conclusion}

In this chapter, we study various models for the one-shot exchange market problem, prove that all of these problems are NP-hard and propose several heuristic and approximation algorithms for these problems. We analyzed

the approximation algorithms by showing some worst-case approximation guarantees.

In the deterministic case, we motivated simple market exchange where only exchanges in the form of swaps are acceptable. While for a very similar kidney exchange problem, this is solvable in polynomial time, we proved that this is NP-complete in our case. When longer exchange cycles are allowed, NP-completeness extends to our setting. We also proposed a probabilistic exchange market problem where there are probabilities associated with a user engaging with another in an exchange and with exchanging one item for another. The hardness results extend to this case.

By leveraging a close connection to weighted $k$-set packing, we developed a heuristic algorithm (Maximal) and approximation algorithms (Greedy, Local Search, and Greedy/Local Search). Using this connection, we showed 
Chapter 2. One-shot Exchange Markets

that known approximation bounds for these algorithms for wighted $k$-set packing carry over to our exchange market problems - both deterministic and probabilistic.

Finally, we conducted a detailed experimentation using synthetically generated data sets, comparing different algorithms on output quality and running time and studying the extent of increase in coverage as maximum cycle length is increased, as well as the impact of data skew on coverage. Our results show that while Maximal does not have provable approximation guarantees, its performance in practice may be comparable to that of the other algorithms. This is interesting given that Maximal is by far the most efficient. 
Algorithm Maximal:

1. Repeat $M$ times:

(a) Construct a graph $G$ from the exchange market instance.

(b) Initialize the set of cycles $\mathcal{B}$ to be the empty set.

(c) While there exists a cycle in graph $G$ :

i. Run a BFS algorithm on graph $G$ starting from a random node to find a cycle $C$ in the graph.

ii. Add cycle $C$ to the set of cycles $\mathcal{B}$.

iii. Remove edges of $C$ and all other conflicting edges of $C$ from graph $G$.

2. Select the maximum weight set among the $M$ sets that are found.

Figure 2.5: Description of the Maximal Algorithm 


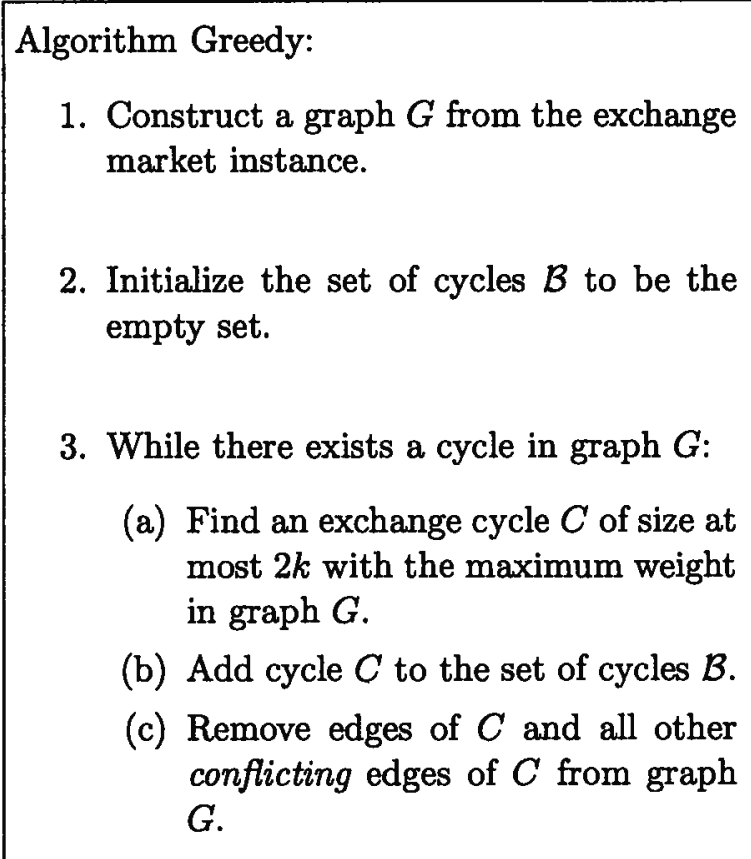

Figure 2.6: Description of the Greedy Algorithm 
Chapter 2. One-shot Exchange Markets

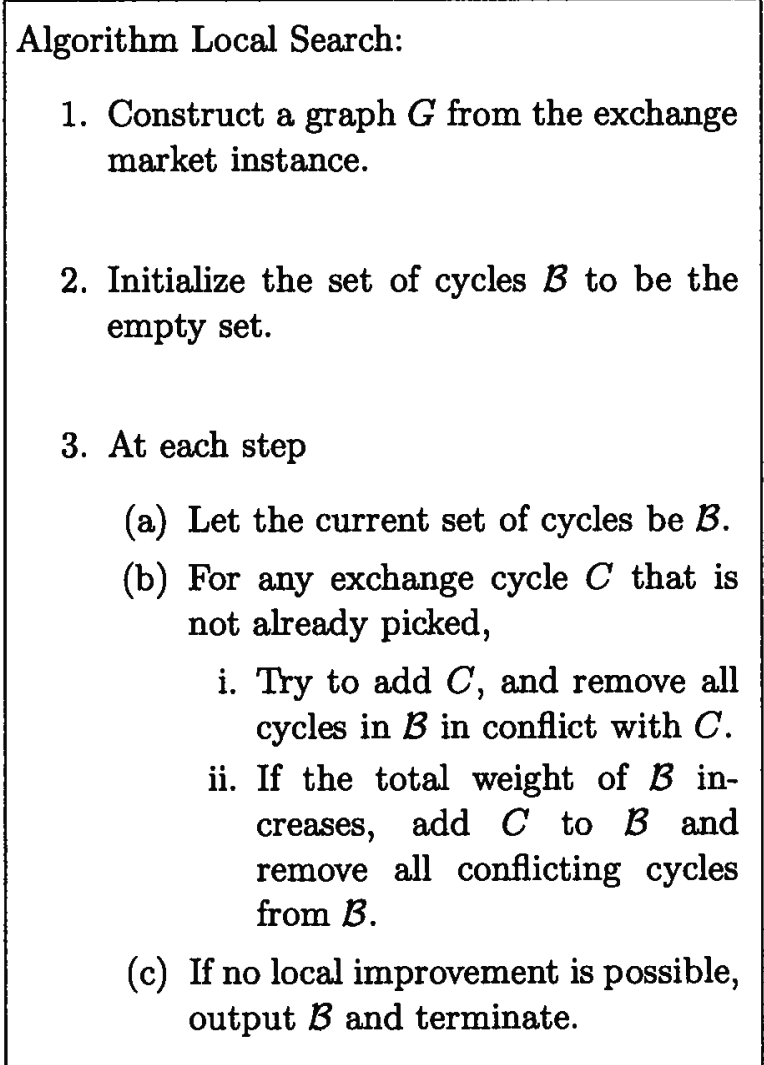

Figure 2.7: Description of the Local Search Algorithm 

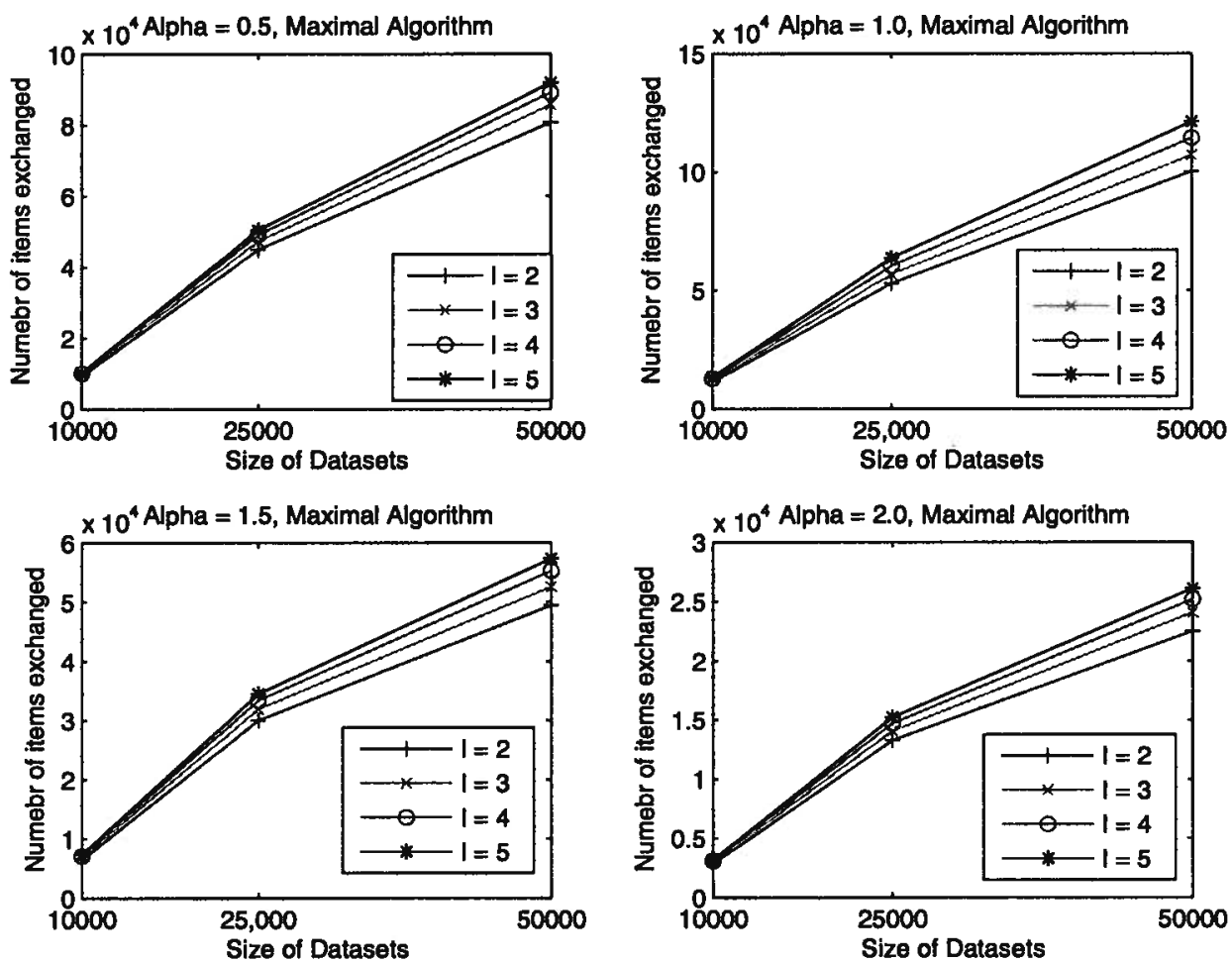

Figure 2.8: Results of the Maximal algorithm on datasets of size 10000 , 25000 and 50000 . 

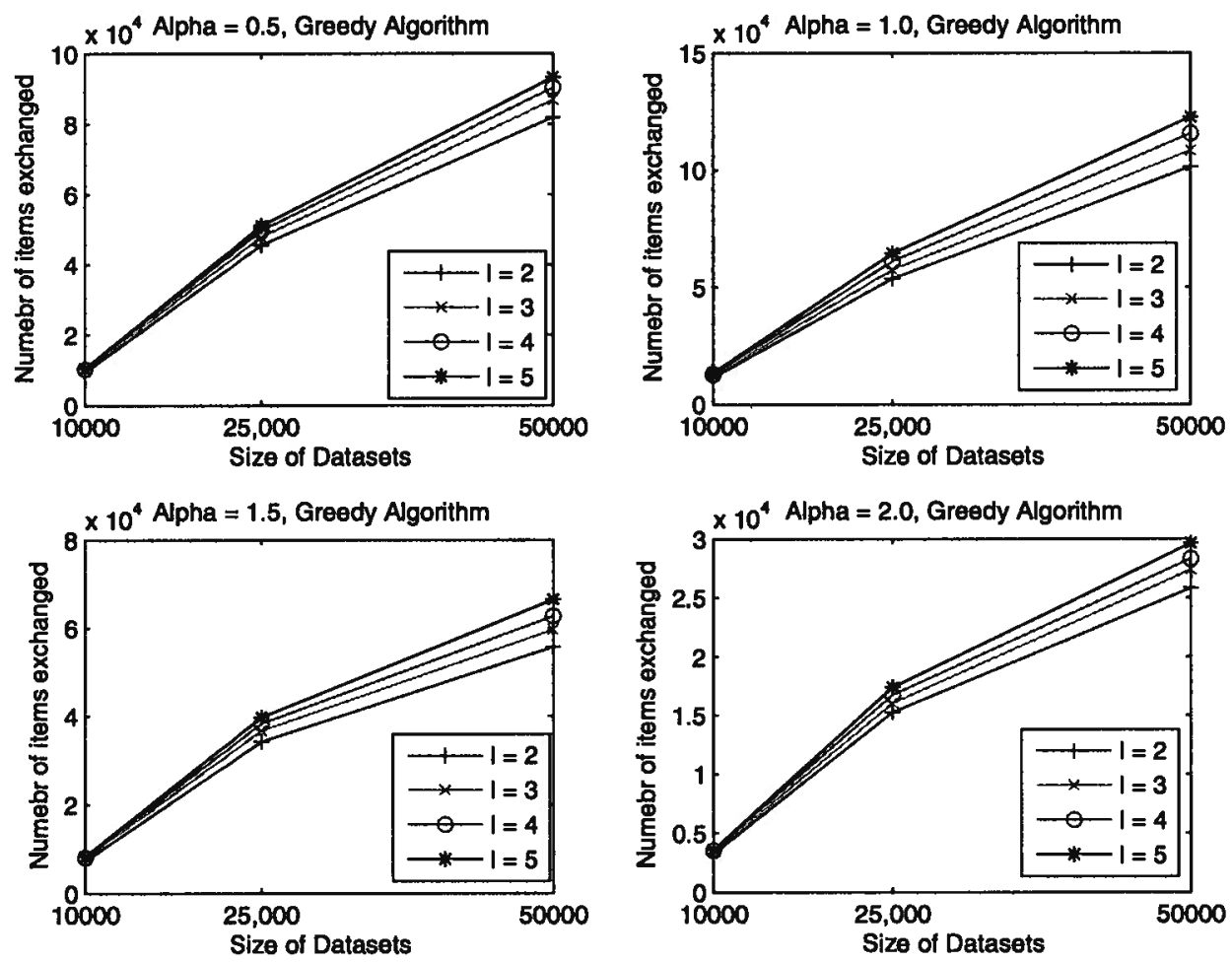

Figure 2.9: Results of the Greedy algorithm on datasets of size 10000, 25000 and 50000 . 
Chapter 2. One-shot Exchange Markets
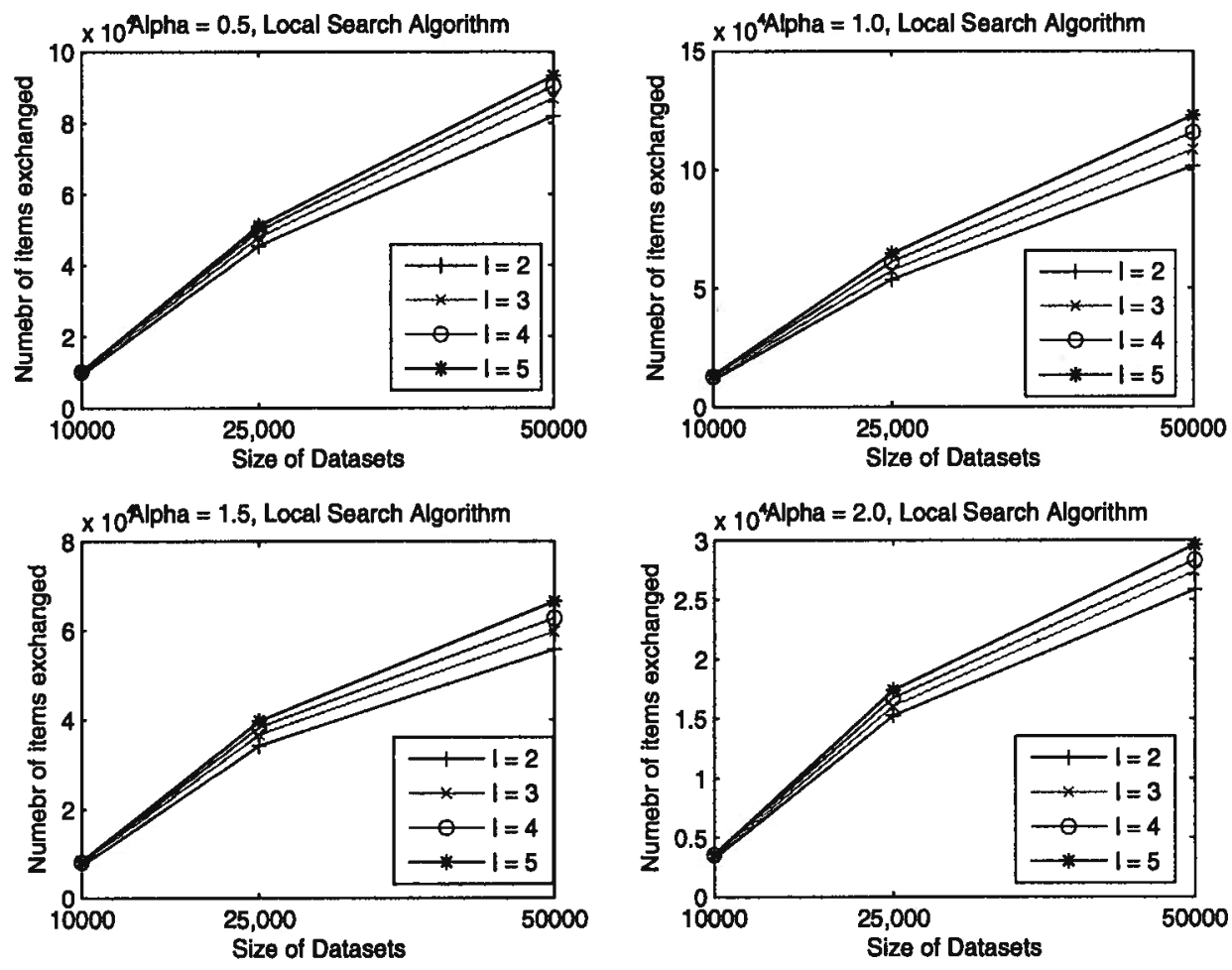

Figure 2.10: Results of the Local Search algorithm on datasets of size 10000, 25000 and 50000 .

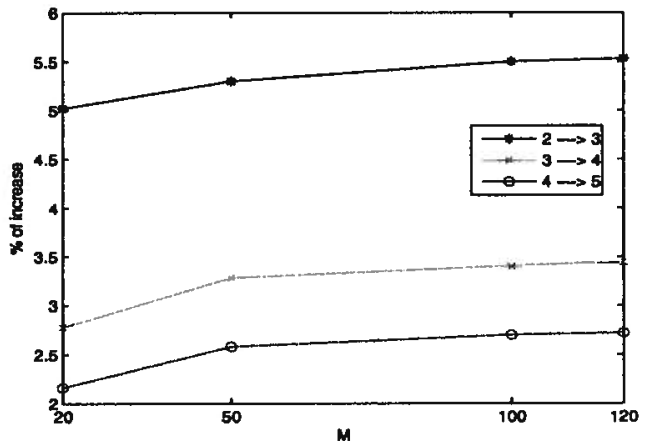

Figure 2.11: Percentage of coverage for different $M$ 's in the Maximal algorithm. 
Chapter 2. One-shot Exchange Markets

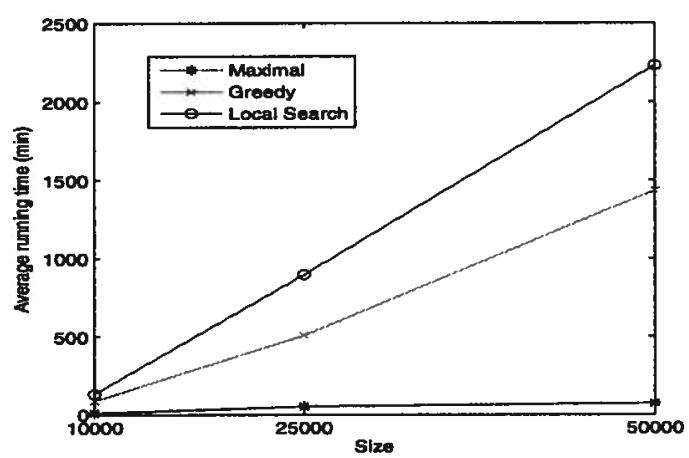

Figure 2.12: Average Running Time of Various Algorithms.

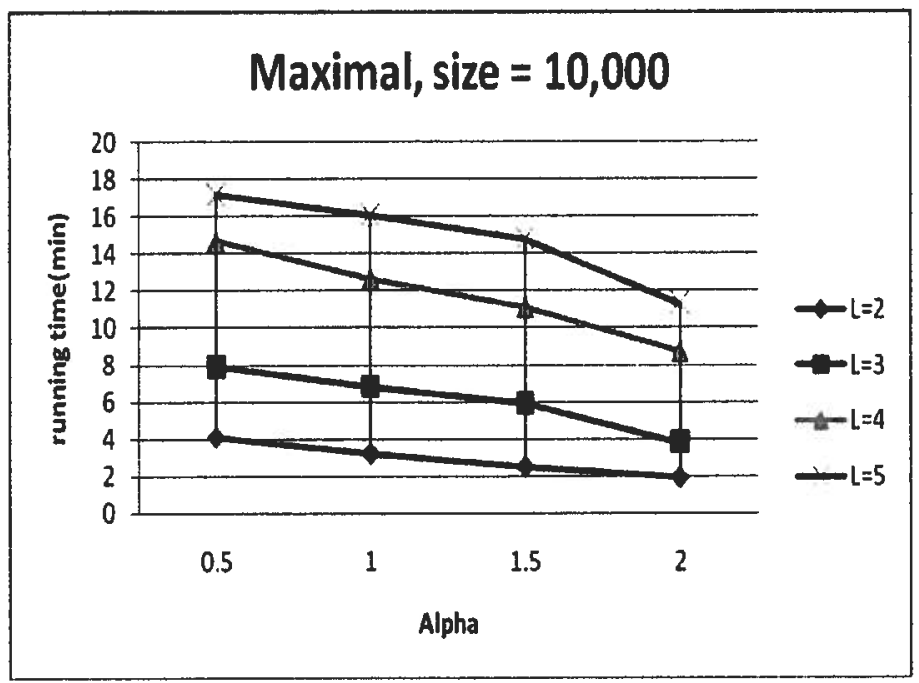

Figure 2.13: Running Time of Algorithm Maximal on dataset of size 10,0000 over various alphas. 
Chapter 2. One-shot Exchange Markets

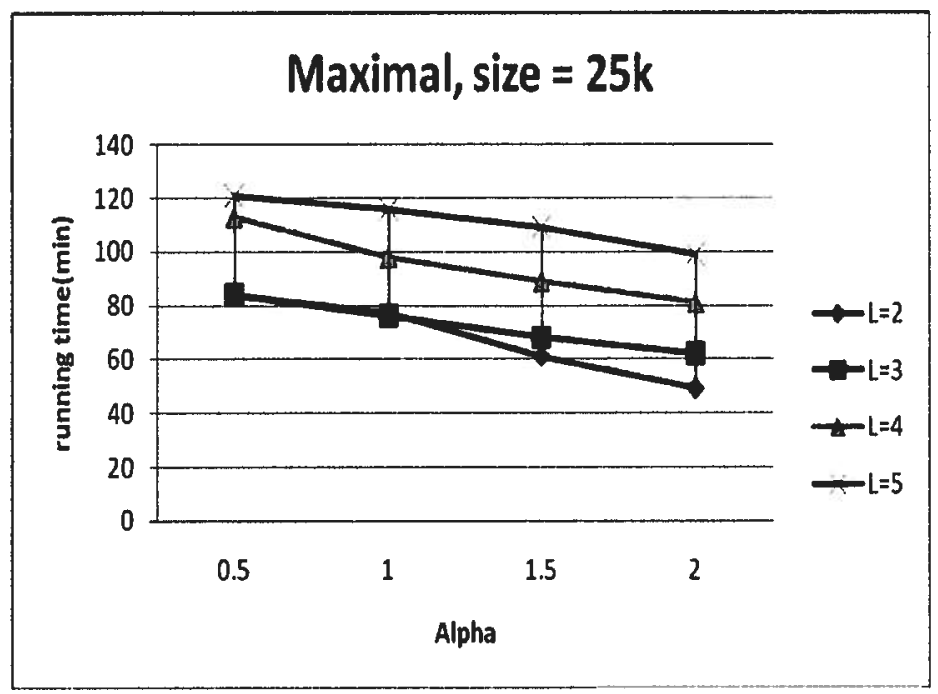

Figure 2.14: Running Time of Algorithm Maximal on dataset of size 25,0000 over various alphas.

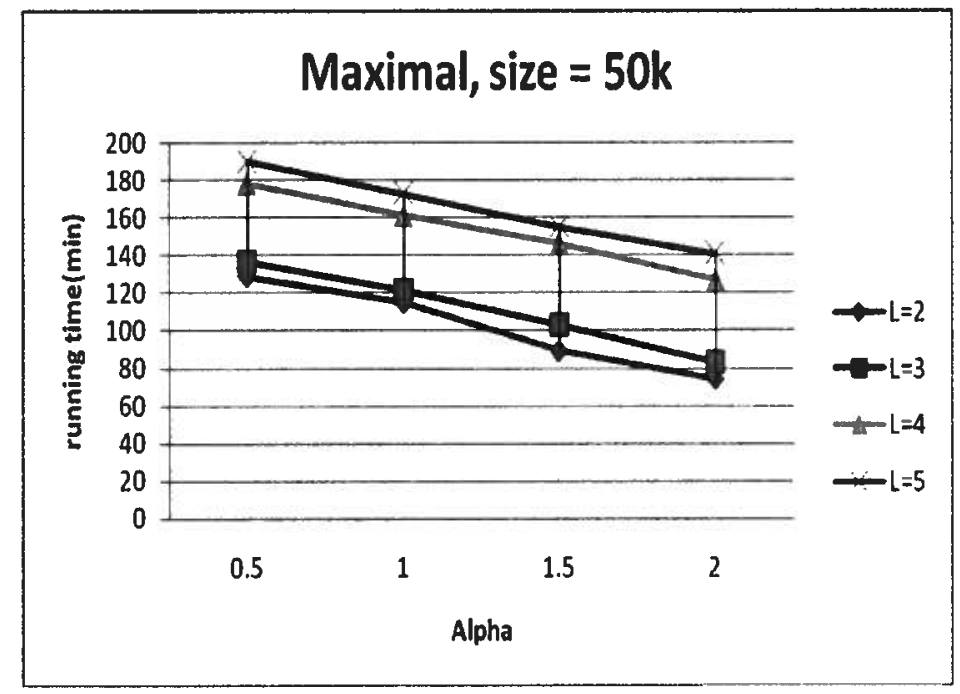

Figure 2.15: Running Time of Algorithm Maximal on dataset of size 50,0000 over various alphas. 
Chapter 2. One-shot Exchange Markets

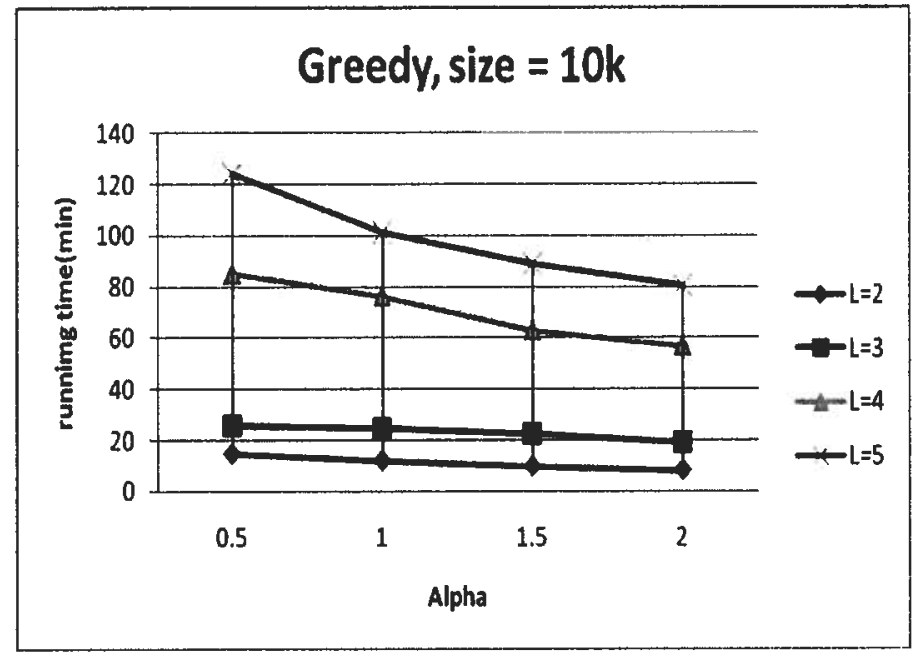

Figure 2.16: Running Time of Algorithm Greedy on dataset of size 10,0000 over various alphas.

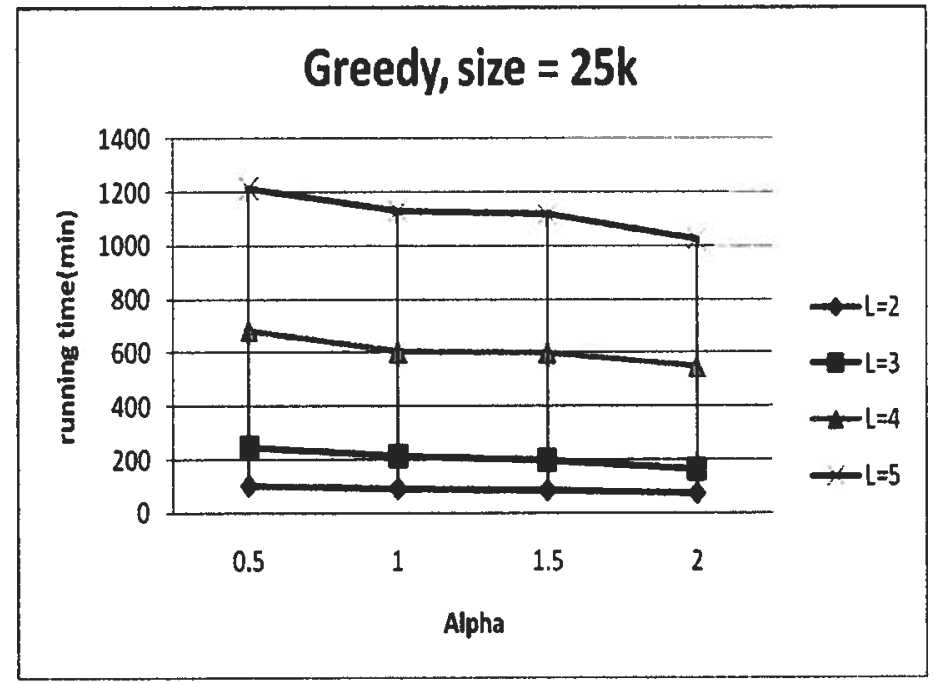

Figure 2.17: Running Time of Algorithm Greedy on dataset of size 25,0000 over various alphas. 
Chapter 2. One-shot Exchange Markets

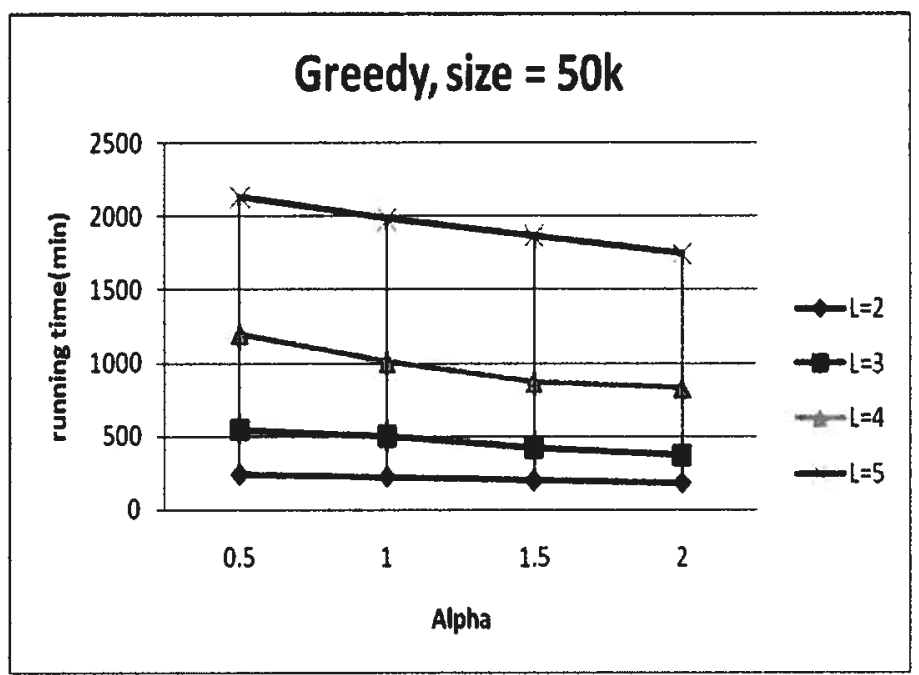

Figure 2.18: Running Time of Algorithm Greedy on dataset of size 50,0000 over various alphas.

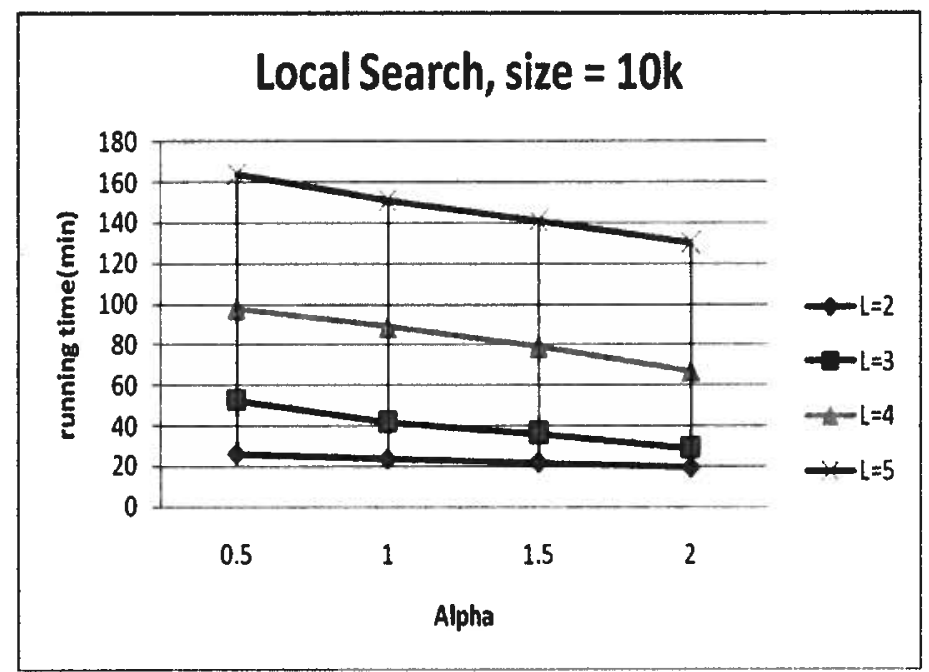

Figure 2.19: Running Time of Algorithm Local Search on dataset of size 10,0000 over various alphas. 
Chapter 2. One-shot Exchange Markets

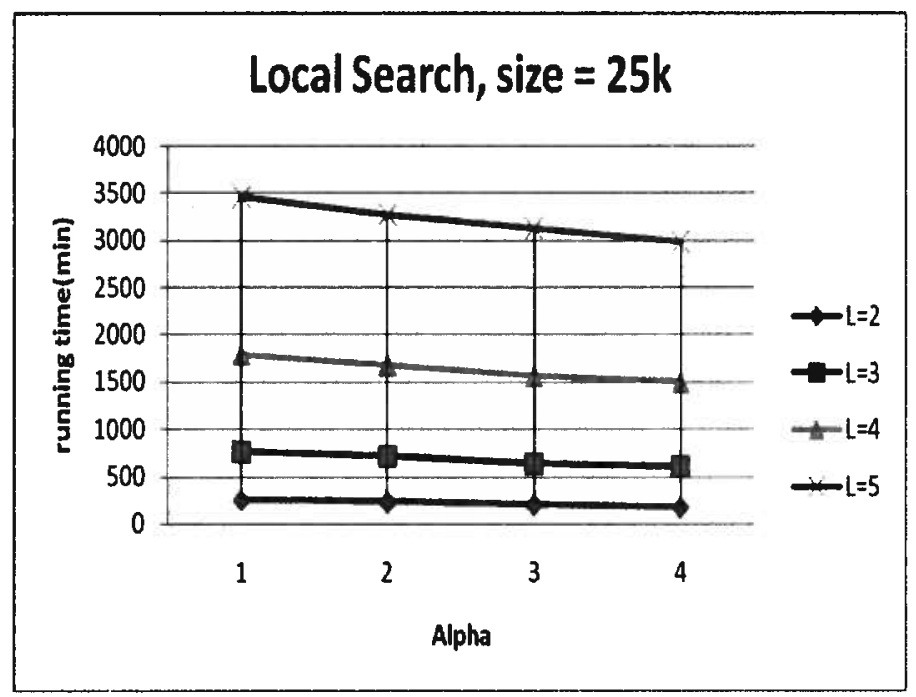

Figure 2.20: Running Time of Algorithm Maximal on dataset of size 25,0000 over various alphas.

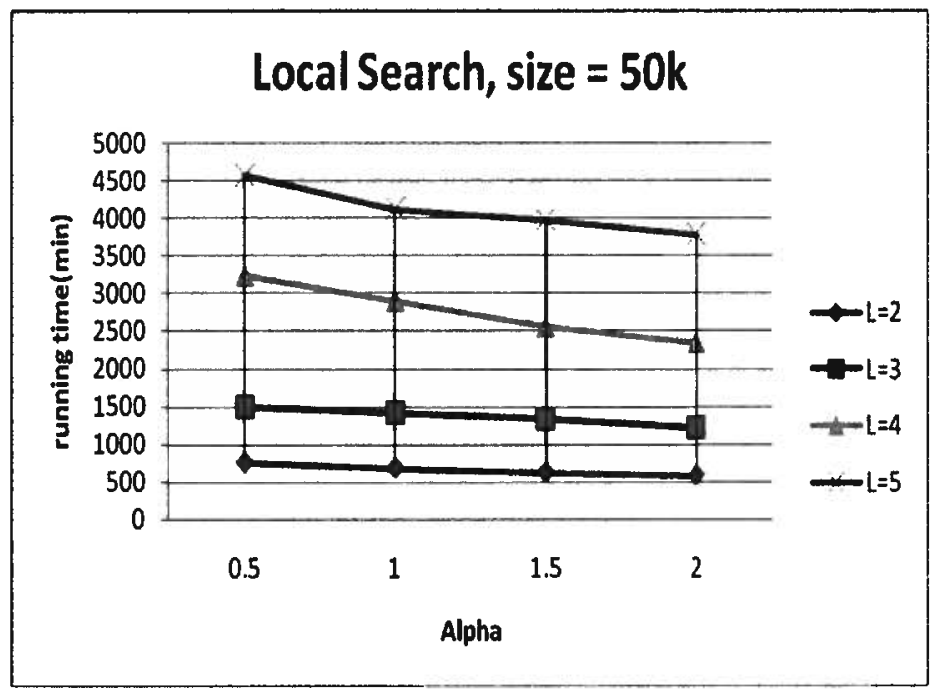

Figure 2.21: Running Time of Algorithm Maximal on dataset of size 50,0000 over various alphas. 


\section{Chapter 3}

\section{Online Exchange Markets}

\section{Over Time}

\subsection{Introduction}

In Chapter 2, we considered online exchange markets under deterministic and probabilistic models. The objective in those one-shot models was to maximize the weight of cycles where weight of a cycle could either be defined as the number of items/users involved in an exchange cycle (in the SimpleMarketProblem and CycleMarketProblem) or the expected number of items/users involved in the transaction in the ProbMarektProblem.

In this chapter we are considering online exchange markets over time and designate following objectives as well as the objective to maximize the expected number of items exchanged.

- Maximizing the number (or the total value) of items exchanged in the market.

- Minimizing the average waiting time of users in the system.

- Maximizing fairness in the system, where by fairness we mean to give 
higher priority in receiving items to users who contribute more to the system by sharing more valuable items.

In this chapter we observe that introducing virtual points and a credit system can decrease the average waiting time by a large margin and also can help in maximizing fairness in the system. In order to implement a complete credit system and virtual points, we need to take into account the following characteristics:

1. Participation: Motivating users for participation in the system by giving more active users more items.

2. Providing Popular items: Motivating users to provide more popular items by giving more credit to users who provide more popular items, where an item is more popular if it appears in more wish lists.

3. Encouraging early participation: Motivating users to participate in the system as early as possible.

4. Fairness: Assigning items to users in the most possible fair way, i.e, giving items to people who have already collected more credit.

\subsection{Related Work}

Related work to this chapter can be classified in the following categories:

\subsubsection{Scheduling Problems}

The problems in scheduling theory that are related to our problem are: 
Minimum makespan scheduling: Given processing times for $n$ jobs, $p_{1}, p_{2}, \ldots, p_{n}$, and an integer $m$, find an assignment of the jobs to $m$ identical machines so that the completion time (makespan) is minimized.

Minimum makespan scheduling problem is NP-hard as we can reduce 2-PARTITION [15] to minimum makespan scheduling on two machines. Horowitz and Sahni [21] give an FPTAS for the variant of the problem when there are a constant number of machines. (An algorithm $A$ is said to be fully polynomial time approximation scheme (FPTAS) if for each $\epsilon>0$ the running time of $A$ is bounded by a polynomial in the size of instance $I$ and $1 / \epsilon$.) The minimum makespan problem is strongly NP-hard for arbitrary $m$ by a reduction from 3-PARTITION [15]. Thus, there cannot exist an FPTAS for the minimum makespan problem in general, unless $\mathrm{P}=\mathrm{NP}$. Hochbaum and Shmoys [19] give a PTAS $((1+\epsilon)$-approximation algorithm which runs in polynomial time for any fixed $\epsilon$ ) for the problem. (An algorithm $A$ is said to be polynomial time approximation scheme (PTAS) if for each $\epsilon>0$ the running time of $A$ is bounded by a polynomial in the size of instance $I$.)

Scheduling on Unrelated Parallel Machines: This problem is a generalization of the minimum makespan scheduling, where the machines are not identical and a job has different processing times on different machines.

Lenstra, Shmoys, and Tardos [23] give a 2-approximation for this version. They also proved that it is not possible to approximate it within a factor $(3 / 2-\epsilon)$ for any $\epsilon>0$, unless $\mathrm{P}=\mathrm{NP}$. Other generalizations include, considering precedence constraints in the scheduling of jobs. Lam and Sethi [27] give a $(2-2 / n)$-approximation for the version where each job has a unit 
processing time.

We use the above problems to prove NP-hardness of the fairness maximization problem 3.6.

\subsubsection{Fairness Maximization in P2P Systems}

The first Peer-to-Peer (P2P) networks were based on the philanthropic behavior of the peers. However, newer P2P applications such as BitTorrent incorporate some kind of incentive mechanism to award sharing peers. One of the main reasons of the recent success of peer to peer (P2P) file sharing systems such as BitTorrent is its built-in tit-for-tat mechanism. For example, a typical Bit- Torrent client uploads to four of its neighbors from which it receives the most download. The intuition behind such a design is that it will encourage upload and lead to a fair and efficient allocation of bandwidth among the peers. In [40] the authors explore if the bandwidth allocation converges when all the users adopt the above strategy. Such a dynamic process is intuitively fair as each node returns more to whoever sent it more. The authors prove that the bandwidth allocation converges to a market equilibrium. In other words, simple as this appears to be, it indeed leads to an efficient allocation of bandwidth in the market equilibrium sense.

The P2P systems mentioned above are different from our system. Users in those systems don't have lists, and the systems do not generate recommendations. Moreover, points and credit system are not defined explicitly. 


\subsection{Model}

Consider a set of users $U$ and a set of items $I$. User $u \in U$ owns a set of items (a.k.a. item list) $S_{u}$, and requires a set of items (a.k.a. wish list) $W_{u}$. Each item $i \in I$ has a value "popularity" $P_{i}$ corresponding to it, which is the number of times it appears in all users' wish lists.

In our proposed system, the algorithm is run periodically and the set of potential feasible exchanges are discovered. Users involved in these potential exchanges are informed through email and/or their account will be updated. If they are interested in performing the transaction, they will get in touch with the other users. Otherwise they will withdraw the transaction and all other involved users will be informed. In any case when the transaction is done the exchanged items are removed from the item lists and wish lists, and the system will be updated.

The system is dynamic meaning that it changes with time. The possible changes are:

- a new user joins the network.

- a user leaves the network.

- an item is removed from a user's wishlist or itemlist: either of these changes may be due to a transaction done or the user's interests changed.

- an item is added to a user's wishlist or itemlist.

These changes imply that the set of feasible exchanges will also change by 
time. Thus, the system runs the algorithm to find the set of new exchanges that have become available periodically (as an example: once a week).

\subsection{Minimizing Average Waiting Time}

In this section, we observe that by introducing "virtual points" in the system, we can decrease the average waiting time of users by a large margin. To implement this idea, we give virtual points to a user for giving away their item, and later give them an item for redeeming their points. As an example let's assume that user $u$ has item $i$ in their item list and item $j$ in their wish list. Also, let's assume user $v$ has item $j$ in their item list, however $v$ does not have item $i$ in their wish list. Thus, a swap is not possible. The idea here is that we recommend item $j$ to $u$ and in return give some points to $v$. In this case $u$ does not have to wait until $v$ wishes for $i$ or a feasible swap comes up. We will elaborate on a credit system that implements exchanging virtual points in the next section. It can be easily seen that, in the worst case, using virtual points can decrease the average waiting time of users by a large margin. In the following, we formally show that introducing points can decrease the expected waiting time, even assuming that all users arrive in a random order.

Theorem 3 Consider an exchange market consisting of $n$ users, and $n$ items. Assume that users put their items at $n$ time steps $T=1,2, \ldots, n$, and we are allowed to assign cycles of size $k$. Let $A$ be the average waiting time of users in a system without points and $B$ be the average waiting time of users in a system with virtual points. There are instances in which in the 
worse case, $A \geq(k-1) B$. Moreover, assuming that users put their items a random permutation, there are instance in which in expectation $A=\frac{2 k}{k+1} B$.

Proof: Assume that we can assign cycles of size at most $k$, and let $p=\frac{n}{k}$. Consider $n$ users in the system with their item lists and wish lists as follows: a cycle of size $k$ among users can be formed by having $k$ users $u_{1}, \ldots, u_{k}$ such that for $1 \leq j<n$, user $u_{j}$ has item $i_{j}$ in her item list and item $i_{j+1}$ in her wish list and user $u_{n}$ has item $i_{n}$ in her item list and $i_{1}$ in her wish list. Clearly, this instance has admits $p=\frac{n}{k}$ exchange cycles of size kof. At the beginning, users have empty item lists and will add items in a random order to their item lists. At the end of the process, user $u_{j}$ for $1 \leq j \leq n$ will have item $i_{j}$ in her item list. Starting from empty item lists, we assume that items become available. In other words, the wish lists will be posted at the beginning (time $T=0$ ), and items arrive on times $T=1, T=2, T=3, \ldots, T=n$, and they are all required in order to complete $p=\frac{n}{k}$ cycles of length $k$.

In the case that we use virtual points in the system, as soon as an item arrives, it will be given to the user that has that item on her wish list. Therefore the waiting times would be $1,2, \ldots, n$,

$$
B=\sum_{i=1}^{n} i=\frac{n(n+1)}{2}=\frac{n^{2}}{2}+\frac{n}{2}
$$


In the worst case, all $\frac{n}{k}$ cycles finish in the last $k$ steps of the process. Thus, in the worst case,

$$
A=k \times \sum_{i=n-\frac{n}{k}+1}^{n} i=k\left(\frac{n(n+1)}{2}-\frac{n(n+k)}{2 k^{2}}\right)=\frac{n(n+1) k^{2}-n(n+k)}{2 k} \geq(k-1) B
$$

Now, we show that assuming that the items arrive in a random order, in expectation, $A \geq \frac{2 k}{k+1} B$. We compute the expected waiting time of users both in the case that we use virtual points in the system, and in the case we do not. The expected value of $B$ is the same as in the previous case, i.e., $B=\frac{n(n+1)}{2}$. Now let's consider the case that we do use virtual points in the system. Let $C_{i}$ be a random variable showing the time that cycle $i$ becomes completed. We also know that the expected waiting time of all cycles being completed is the same:

$$
E\left[C_{1}\right]=E\left[C_{2}\right]=\ldots=E\left[C_{p}\right]
$$

The waiting time in this case would be:

$$
\sum_{i=1}^{p} k C_{i}=k \sum_{i=1}^{p} C_{i}
$$

By linearity of expectations, the expected waiting time is equal to

$$
k \sum_{i=1}^{p} E\left[C_{i}\right]=k p E\left[C_{i}\right]=n E\left[C_{i}\right]
$$


Now, we compute each $E\left(C_{i}\right)$ as follows:

$$
\begin{aligned}
E\left[C_{i}\right] & =\sum_{j=k}^{n} \operatorname{Pr}\left(C_{i}=j\right) j \\
& =\sum_{j=k}^{n} \frac{\left(\begin{array}{l}
j-1 \\
k-1
\end{array}\right) j}{\left(\begin{array}{l}
n \\
k
\end{array}\right)} \\
& =\frac{1}{\left(\begin{array}{l}
n \\
k
\end{array}\right)} \sum_{j=k}^{n}\left(\begin{array}{l}
j-1 \\
k-1
\end{array}\right) j \\
& =\frac{k}{\left(\begin{array}{l}
n \\
k
\end{array}\right)} \sum_{j=k}^{n}\left(\begin{array}{c}
j \\
j-k
\end{array}\right) \\
& =\frac{k}{\left(\begin{array}{c}
n \\
k
\end{array}\right)} \sum_{i=0}^{n-k}\left(\begin{array}{c}
k+i \\
i
\end{array}\right) \\
& =\frac{k}{\left(\begin{array}{l}
n \\
k
\end{array}\right)}\left(\begin{array}{l}
n+1 \\
n-k
\end{array}\right) .
\end{aligned}
$$

Therefore, the total expected waiting time is

$$
A=E[C]=n E\left(C_{i}\right)=n \frac{k(n+1)}{(k+1)}=\frac{k n(n+1)}{(k+1)} .
$$

As a result, the ratio between the expected waiting time with and without virtual points is $\frac{A}{B}=\frac{2 k}{k+1}$.

\subsection{A Credit System}

In this section, we design a credit system that takes into account the properties that we mentioned earlier. We design a credit system in which each user $i$ collects credit for providing items, and can redeem credit points to receive some items. Let $C_{i}(t)$ be the credit of user $i$ at time $t$. Each item $j$ 
has a credit score $P_{j}$ which can be a function of the popularity of item $j$. Initialization: We can initialize the credit of each new user in the system to zero or a small positive value.

Updating Rules: Consider a transaction $T$ at time $t$ in which a user $i$ gives an item $j$ to a user $i^{\prime}$, i.e, item $j$ is in item list of $i$ and in wish list of $i^{\prime}$. In the following we describe how to choose transaction $T$ and how to update the credit of each user after transaction $T$ happens to satisfy the desired properties discussed in the previous section.

- Participation: To satisfy this property, we make sure that we choose a user $i^{\prime}$ to get the item such that $C_{i^{\prime}}(t)$ is large (perhaps the largest) among all users who has item $j$ in their wish list.

In general, after transaction $T$ happens, we update $C_{i}(t+1)=C_{i}(t)+$ $P_{j}(t)$ and $C_{i^{\prime}}(t+1)=C_{i^{\prime}}(t)-P_{j}(t)$.

- Providing Popular items: To satisfy this property, we set $P_{j}(t)$ to be a function of the number of times that item $i$ appears in the wish lists.

- Encouraging early participation: To satisfy this property, we update the scores of users after each time step as follows: $C_{k}(t+1)=$ $C_{k}(t) *(1+\epsilon)$ where $\epsilon$ is a small interest rate, i.e, $\epsilon=0.001$. This will encourage users to participate in the system as early as possible.

- Fairness: To satisfy fairness in an online way, we should minimize the maximum credit of a user at each step. As a result, in order to satisfy fairness, we wait for a period of time (e.g., a day) for users to post 
new items in their item list (and wish lists), and then assign the items to other users in the most fair manner. we will formalize this problem formally in the next section.

\subsection{Maximizing Fairness}

In this section, we can formalize the fairness problem for assigning all new items throughout a fixed time window (e.g., one day) to a set of users. Consider a time step $t$ at which each user has a current credit $C_{i}{ }^{2}$ Let $S_{i}$ be the new set of items in the item list of users during the last time day. Let $W_{i}$ be the wish list of user $i$, and $P_{j}$ be the current value for item $j$, i.e, if we give $j \in W_{i}$ to user $i, C_{i}(t+1)=C_{i}-P_{j}$. We want to assign items in sets $S_{i}$ to users such that:

- Each item in each item list $S_{i}$ is assigned to at most one user.

- If item $j \in S_{i}$ is given to user $i^{\prime}$, then $j \in W_{i^{\prime}}$.

- Each user $i$ gets at most one copy of item $j \in W_{i}$ in his/her wish list.

The goal is to find such assignment that minimizes the maximum credit in the next step, i.e, $\min \max _{i} C_{i}(t+1)^{3}$.

The only issue with the above formulation is that it is possible that a user $k$ with a large credit provide some new items and not assigning these items may give a better solution since if user $k$ in this situation provides

\footnotetext{
${ }^{2}$ We drop the parameter $t$ as it is clear from the context.

${ }^{3}$ The ultimate goal is to find a fair assignment that minimizes the maximum credit, and subject to this, we minimize the second maximum, and so on, but for the sake of simplicity and as a first attempt, we look at the problem of minimizing the maximum credit.
} 
more items, his score increases even more, and as a result the maximum score increases. Here there is a trade off between the expected waiting time of users (or similarly the number of items exchanged in the system) and the above fairness property. A middle-way solution to take into account the aforementioned trade off is to say that we want to find an assignment of items in $S_{i}$ 's to users such that the assignment is maximal and it minimizes the maximum credit, i.e, after assigning items from item lists to wish lists, no other item can be assigned from item lists to wish lists of users.

Theorem 4 The fairness maximization problem is NP-complete.

Proof: We give a reduction from a scheduling problem with restricted assignment $\left(B \| C_{\max }\right)$. In a scheduling problem with restricted assignment $\left(B \| C_{\max }\right)$, we have $n$ jobs and $m$ machines, and each job $j$ has a processing time $P_{j}$ and can be scheduled on a subset $U_{j}$ of machines. Given an assignment of jobs to machines, where each job $j$ is assigned to a machine in $U_{j}$, the load of machine $i, L_{i}$ is the sum of processing time of jobs assigned to $i$. The goal is to assign jobs to machines to minimize the makespan or the maximum load of each machine, i.e., min assignment $\max _{i} L_{i}$.

There is a clear reduction from the above problem to the max fairness problem. Here is the reduction: Given an instance of $B \| C_{\max }$, we put a user for each of the $n$ jobs, and also we put a user for each machine $i$ (in total, we have $m+n$ users). For user $i$ corresponding to machine $i$, we have credit $C_{i}(t)=C_{i}=0$, with empty wish list $\left(W_{i}=\emptyset\right)$ and a large item list $S_{i}$ with all jobs that can be scheduled on machine $i$ in it (i.e., $S_{i}=\left\{j \mid i \in U_{j}\right\}$ ). For user $j$ corresponding to job $j$ in the instance, we 
put the initial credit $C_{j}=P_{j}$, a wish list $W_{j}=\{j\}$ and an empty item list. It is easy to see that an assignment of jobs to machines in the original $B \| C_{\max }$ problem correspond to an exchange of items in the new instance. In particular, the load of machine $i$ is equal to the credit of user $i$ in the new instance. It is clear to see that the problem of minimizing makespan in the original instance of $B \| C_{\max }$ is equivalent to the fairness problem in this new instance, i.e, in order to minimize the maximum load of machines in $B \| C_{\max }$, we should minimize the maximum credit.I

As also mentioned in Section 3.2 the $B \| C_{\max }$ problem is NP-hard and not approximable within any constant factor better than $3 / 2$ [23]. $B \| C_{\max }$ and the more general version in which the processing time of each job on each machine is different (i.e, instead of $P_{j}$, we have $P_{i j}$ ), is approximable within a factor 2 .

Thus, the above reduction shows that the fairness problem is NP-hard, and moreover, since the objective functions of the two problems are the same, if we can optimize the fairness problem within a factor better than $3 / 2$, then we can optimize the $B \| C_{\max }$ scheduling problem within a factor better than $3 / 2$.

In Section 3.8 we prove that our problem is not approximable within any multiplicative factor.

\subsection{A Linear Programming Formulation}

In this section, we develop an integer linear programming formulation of the fairness problem. For a triple $\left(i, i^{\prime}, j\right)$ where $j \in S_{i}$ and $j \in W_{i^{\prime}}$, let $x_{i i^{\prime} j}=1$ 
if we assign item $j$ from $i$ 's item list to user $i^{\prime}$, and $x_{i i^{\prime} j}=0$ otherwise. Also, we let variable $t_{i}$ be a variable that corresponds to the credit of user $i$ after the assignment of this step, and variable $t$ be the maximum of credits, or $\max _{i \in U} t_{i}$. Finally, for easy of notation, we let $U$ be the set of users and $I$ be set of items.

$\min$

$$
\begin{aligned}
& t \geq t_{i} \quad \forall i \in U \\
& t_{i}=C_{i}+\sum_{j \in S_{i}} \sum_{i^{\prime}: j \in W_{i^{\prime}}} x_{i i^{\prime} j} P_{j}-\sum_{j \in W_{i}} \sum_{i^{\prime}: j \in S_{i^{\prime}}} x_{i^{\prime} i j} P_{j} \quad \forall i \in U \\
& \sum_{i^{\prime}: j \in W_{i^{\prime}}} x_{i i^{\prime} j} \leq 1 \quad \forall i \in U, j \in S_{i} \\
& \sum_{i^{\prime}: j \in S_{i^{\prime}}} x_{i^{\prime} i j} \leq 1 \quad \forall i \in U, j \in W_{i} \\
& x_{i i^{\prime} j}+\sum_{i^{\prime \prime}: j \in S_{i^{\prime \prime}, i^{\prime \prime} \neq i}} x_{i^{\prime \prime} i^{\prime} j}+\sum_{i^{\prime \prime}: j \in W_{i^{\prime \prime}, i^{\prime \prime} \neq i^{\prime}}} x_{i i^{\prime \prime} j} \geq 1 \quad \forall i, i^{\prime} \in U, j \in S_{i} \cap W_{i^{\prime}} \\
& x_{i i^{\prime} j} \in\{0,1\} \quad \forall i \forall j \in S_{i} \forall i^{\prime}: j \in W_{i^{\prime}}
\end{aligned}
$$

The first inequality indicates that $t$ is the maximum credit of users. The second inequality quantifies the credit of user $i$ after the assignment. The third inequality models the constraint that each item $j$ in the item list of user $i$ can be assigned to somebody at most once, and the forth inequality models the fact that each item $j$ in the with list of user $i$ can be received by user $i$. Finally, the fifth inequality models the maximality of the solution, i.e, fact that if user $i$ does not give an item $j \in W_{i^{\prime}}$ to user $i^{\prime}$, then either user $i$ gives $j$ to somebody else, or user $i^{\prime}$ gets it from somebody else.

The LP relaxation is as follows: 
$\min$ $t$

$$
\begin{array}{cl}
t \geq t_{i} & \forall i \in U \\
t_{i}=C_{i}+\sum_{j \in S_{i}} \sum_{i^{\prime}: j \in W_{i^{\prime}}} x_{i i^{\prime} j} P_{j}-\sum_{j \in W_{i}} \sum_{i^{\prime}: j \in S_{i^{\prime}}} x_{i^{\prime} i j} P_{j} & \forall i \in U \\
\sum_{i^{\prime}: j \in W_{i^{\prime}}} x_{i i^{\prime} j} \leq 1 & \forall i \in U, j \in S_{i} \\
\sum_{i^{\prime}: j \in S_{i^{\prime}}} x_{i^{\prime} i j} \leq 1 & \forall i \in U, j \in W_{i} \\
x_{i i^{\prime} j}+\sum_{i^{\prime \prime}: j \in S_{i^{\prime \prime}, i^{\prime \prime} \neq i}} x_{i^{\prime \prime} i^{\prime} j}+\sum_{i^{\prime \prime}: j \in W_{i^{\prime \prime}, i^{\prime \prime} \neq i^{\prime}}} x_{i i^{\prime \prime} j} \geq 1 & \forall i, i^{\prime} \in U, j \in S_{i} \cap W_{i^{\prime}} \\
0 \leq x_{i i^{\prime} j} \leq 1 & \forall i \forall j \in S_{i} \forall i^{\prime}: j \in W_{i^{\prime}}
\end{array}
$$

This LP is similar to the LP for the scheduling problem but has a minus sign in the inequality for $T_{i}$. It would be interesting to study this LP and see if a similar approach works. In particular, it would be nice to study the basic feasible solutions of this LP.

\subsection{A Strong Inapproximability Result}

In this section, we show that the fairness maximization problem is not approximable within any factor.

Theorem 5 The problem of fairness optimization is not approximable within any multiplicative factor.

Proof: We show that if we can approximate this problem within any factor, we can solve an NP-complete problem, i.e., the partitioning problem. In an instance of the partitioning problem, we are given $n$ numbers 
$a_{1}, a_{2}, \ldots a_{n}$ with the total sum of $B=\sum_{i=1}^{n} a_{i}$, and our goal is to verify if there exists a partition of these number into two sets such that each subset has the total sum of $\frac{B}{2}$. It is known that the partitioning problem is an NPcomplete problem. We say that an instance of the partitioning problem is a YES instance, if there exists a subset of number of total sum $\frac{B}{2}$. Otherwise, the instance is a NO instance.

We give a reduction from the partitioning problem to the fairness problem. In fact, from any instance of the partitioning problem, we construct an instance of the fairness problem in which the optimal solution to the fairness problem is greater than zero if and only if the partitioning problem is a YES instance. Given an instance of the partitioning problem with $n$ numbers $a_{1}, a_{2}, \ldots a_{n}$ where $\sum_{i=1}^{n} a_{i}=B$, we construct an instance of the fairness problem as follows: We put $n$ items and $n+2$ users in the instance of the fairness problem, $n$ users $1,2,3, \ldots, n$ corresponding to $n$ numbers in the partitioning problem, and two extra users $n+1$ and $n+2$ (that get items from the first $n$ users). For each user $i$ where $1 \leq i \leq n$, we put the initial credit $C_{i}=-a_{i}$, wish list $W_{i}=\emptyset$ and item list $S_{i}=\{i\}$. For two users $n+1$ and $n+2$, we put $C_{n+1}=C_{n+2}=B / 2$, empty item lists $S_{n+1}=S_{n+2}=\emptyset$ and $W_{n+1}=W_{n+2}=\{i \mid 1 \leq i \leq n\}$.

Now the optimal solution for the fairness problem is zero if and only if the partitioning instance is a YES instance. The reason is that the only way that the credit of both $n+1$ and $n+2$ goes down to zero if and only if both of these users get a set of items of total credit $\frac{B}{2}$.

More formally, if the partitioning problem is a YES instance, and there exists a set $S_{1}$ of number of total sum $\frac{B}{2}$, then set $S_{1}$ of users can give items 
to user $n+1$ and the rest of users can give items to user $n+2$, and a result the credit of each user in the next step is zero. Conversely, if the optimal resulting credit is zero, it means that both users $n+1$ and $n+2$ got exactly $\frac{B}{2}$ total credit from users $1,2, \ldots, n$. Thus, the set of users who gave an item to $n+1$ construct a subset of numbers with total sum $\frac{B}{2}$ and therefore, the partitioning problem is a YES instance.

The above shows that if we can verify if the optimal solution for the fairness problem is zero or any number above zero, then we can distinguish between the YES and NO instance of the partitioning problem. Therefore, if there exists an $\alpha$-approximation algorithm for the fairness problem, it will output $\alpha \times 0=0$ for an instance of the fairness problem with optimal solution zero. Hence, no approximation algorithm exists for this problem. I

\subsection{Heuristic Algorithms}

Here, we propose two heuristic algorithms to solve the fairness problem; one is a simple fast greedy algorithm, and one is based on randomized rounding of the linear programming formulation discussed in the previous section. The basic idea of the rounding algorithm is a standard method for solving integer linear programs.

\subsubsection{Greedy Algorithm}

A very simple heuristic algorithm is to assign item transfers among users one by one, and at each step find the one item transfer that minimizes the maximum credit after this item transfer. In other words, at each step, we 
examine all possible item transfers for items $j \in S_{i} \cap W_{i^{\prime}}$ for any two users $i$ and $i^{\prime}$, and find the triple $i, i^{\prime}, j$ such that after item transfer, the maximum credit the minimum possible. To do so, we sort the users in the decreasing order of their credit, and try transferring items from users at the beginning of the list.

\subsubsection{An LP-rounding Algorithm}

Consider the linear programming relaxation discussed for the fairness problem. In the integer linear program $x_{i i^{\prime} j}^{*}$ is 1 if use $i$ give an item $j \in S_{i} \cap W_{i^{\prime}}$ to user $i^{\prime}$. Consider a solution $x^{*}$ to this LP. In the LP relaxation, we can interpret the variable $x_{i i^{\prime} j}$ to be the extent at which user $i$ gives item $j$ to user $i^{\prime}$. In other words, we use the value $x_{i i^{\prime} j}^{*}$ as the probability of transferring item $j$ from $i$ to $i^{\prime}$. In order to find a feasible solution, we need to perform the following rounding algorithm.

1. Initialize $\bar{x}=0$.

2. Solve the LP relaxation and find an optimal solution $x^{*}$.

3. For each user $i$ and item $j \in S_{i}$ such that $\sum_{i} \bar{x}_{i i^{\prime} j}=0$,

(a) Let $Z_{i^{\prime} j}=\sum_{i} \bar{x}_{i i^{\prime} j}$.

(b) Let $Y_{i j}=\sum_{i^{\prime}: Z_{i^{\prime} j}=0} x_{i i^{\prime} j}^{*}$.

(c) Among all users $i^{\prime}$ for which $x_{i i^{\prime} j}^{*}>0$ and $\bar{x}_{i i^{\prime} j}=0$,

i. Choose one user $i^{\prime \prime}$ with prob probability $\frac{x_{i i^{\prime \prime} j}^{*}}{Y_{i j}}$.

ii. Set $\bar{x}_{i i^{\prime \prime} j}=1$, and $S_{i}=S_{i}-\{j\}$. 
4. For each item $j$ and each $i^{\prime}$ such that $j \in W_{i^{\prime}}$,

(a) Let set $T_{i^{\prime}}$ be the set of users $i$ for which $\bar{x}_{i i^{\prime} j}$ became one in this round.

(b) If $\left|T_{i^{\prime}}\right|=1$, then for each $i^{\prime \prime} \in T_{i^{\prime}}, x_{i^{\prime \prime} i^{\prime} j}^{*}=0$, and $W_{i^{\prime}}=W_{i^{\prime}} \backslash\{j\}$ (finalize transfer $\left(i^{\prime \prime} i^{\prime} j\right)$ ),

(c) else

i. Choose one user $i^{\prime \prime} \in T_{i^{\prime}}$ with probability $\frac{1}{\left|T_{i^{\prime}}\right|}$.

ii. For each $i \neq i^{\prime \prime}$ and $i \in T_{i^{\prime}}$, set $\bar{x}_{i i^{\prime} j}=0$, and $S_{i}=S_{i} \cup\{j\}$.

iii. For $i^{\prime \prime}$, let $x_{i^{\prime \prime} i^{\prime} j}^{*}=0$, and $W_{i^{\prime}}=W_{i^{\prime}} \backslash\{j\}$ (finalize transfer $\left.\left(i^{\prime \prime} i^{\prime} j\right)\right)$.

5. If any new item transfer was assigned in the last round, go back to 3 to start a new round, otherwise output $\bar{x}$ and terminate.

\subsection{Experimental Evaluation}

We implemented the integer linear program formulation of the fairness maximization problem, linear program formulation of the problem using GNU Linear Program Kit (GLPK), and also the two heuristics: LP-rounding and the greedy heuristic using MATLAB. The experiments were run on a Computer with a $2.40 \mathrm{GHz}$ Intel Core 2 Duo CPU and 3 GB of RAM under Windows Vista.

The goals of our experiments are as follows:

- To examine the quality of the results found by the heuristic algorithms 
since we proved that the fairness maximization problem is not approximable.

- To study the scalability of the heuristic algorithms and also the impact of the parameter $\alpha$.

The LP relaxation discussed in the previous sections gives a polynomialtime computable lower bound on the optimal value of the fairness optimization problem. In fact, we use this lower bound to evaluate the performance of our heuristic algorithms.

\subsubsection{Dataset}

We could not get access to the data of real online exchange applications. Hence, we generated a set of synthetic data. Data generation for the markets over time is similar to what we did in the previous chapter for one-shot exchange markets which was explained in Section 2.6.1.

\subsubsection{Experiments}

The following experiments were performed in order to examine the performance of the heuristic algorithms. We implemented the integer linear programming (ILP) and also the linear programming (LP) formulation of the problem using GLPK and the LP-rounding and the greedy heuristic using MATLAB. We ran our algorithms on three different data sets with 100, 500 and 1000 users. The data were generated with four different values for $\alpha=0.25,0.5,0.75$ and 1 . 


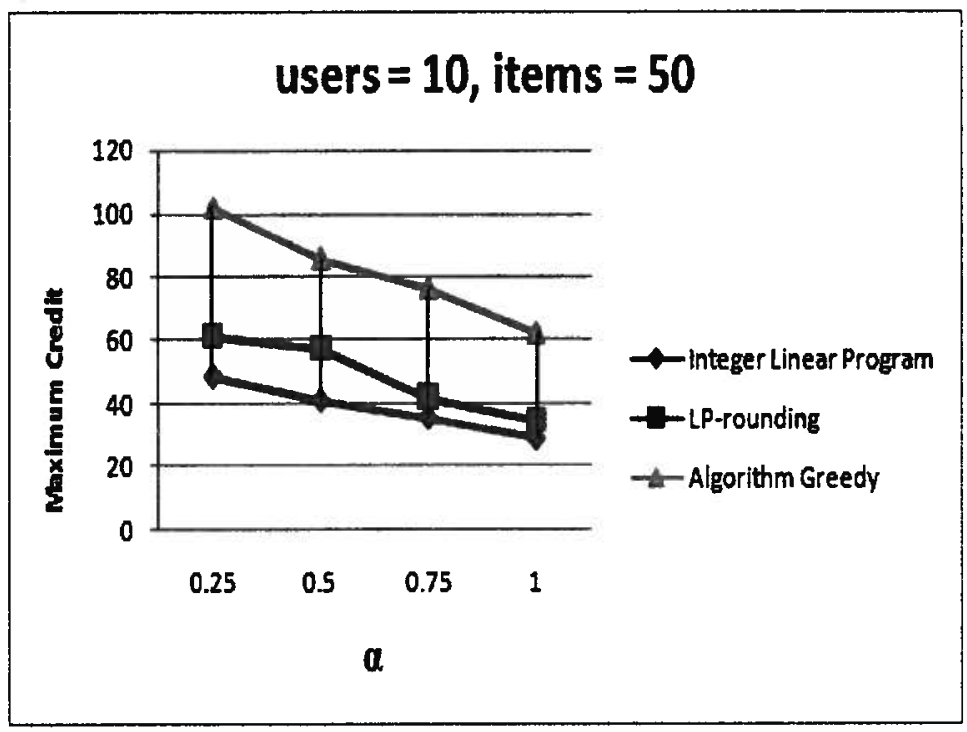

Figure 3.1: Performance of the heuristics compared to the optimal solution given by ILP on dataset of 10 users.

We solved the ILP for two small datasets including 10 users, 50 items and 20 users, 100 items. The ILP gives us the optimal solution however its running time is exponential. We compare the results of the LP-rounding and greedy on same datasets to the optimum. The results are depicted in Figures 3.1 and 3.2 respectively.

Figures 3.3, 3.4, and 3.5 show the results of the LP, LP-rounding and the greedy heuristic for datasets of size 100, 500, and 1000 respectively. The number of total items in each case is 5 times the number of users. The $\mathrm{Y}$ axis indicates the maximum credit, and the $\mathrm{X}$-axis shows $\alpha$ for the different algorithms. We have used the LP results a lower bound on the optimal value. As can be seen, the LP-rounding has a better performance than the other heuristic. Also, we can observe that as $\alpha$ increases (in most cases), 


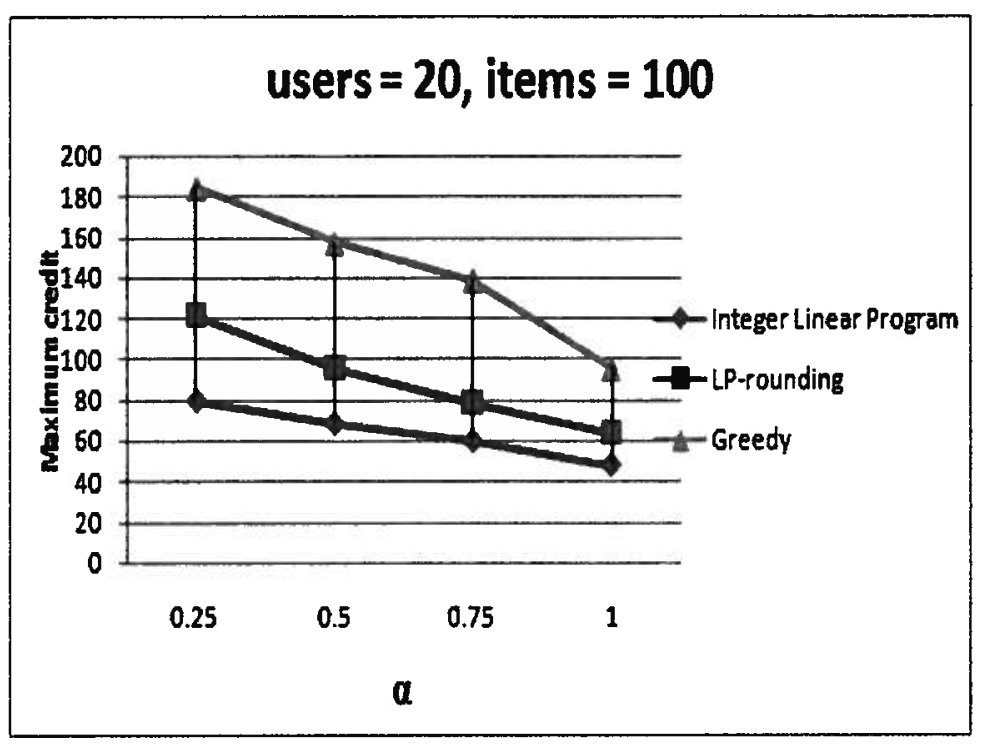

Figure 3.2: Performance of the heuristics compared to the optimal solution given by ILP on dataset of 20 users.

the algorithms' performance also improves.

The running times of the above algorithms are also depicted in Figures 3.6, 3.7, and 3.8 for datasets of size 100,500 , and 1000 respectively. As can be seen, the running time of the greedy algorithm is better than the LProunding heuristic. Moreover, we can see that by increasing $\alpha$, the running time improves.

Another measure that we explored was the number of transactions performed at each time step. It is important that the users stay active and have incentive to share their items, even when they have received an item from their wish list. Figure 3.9 plots the percentage of active users in at six consecutive time windows in a simulation done with 100 users.

As can be seen at the beginning we have the maximum activity. The 
Chapter 3. Online Exchange Markets Over Time

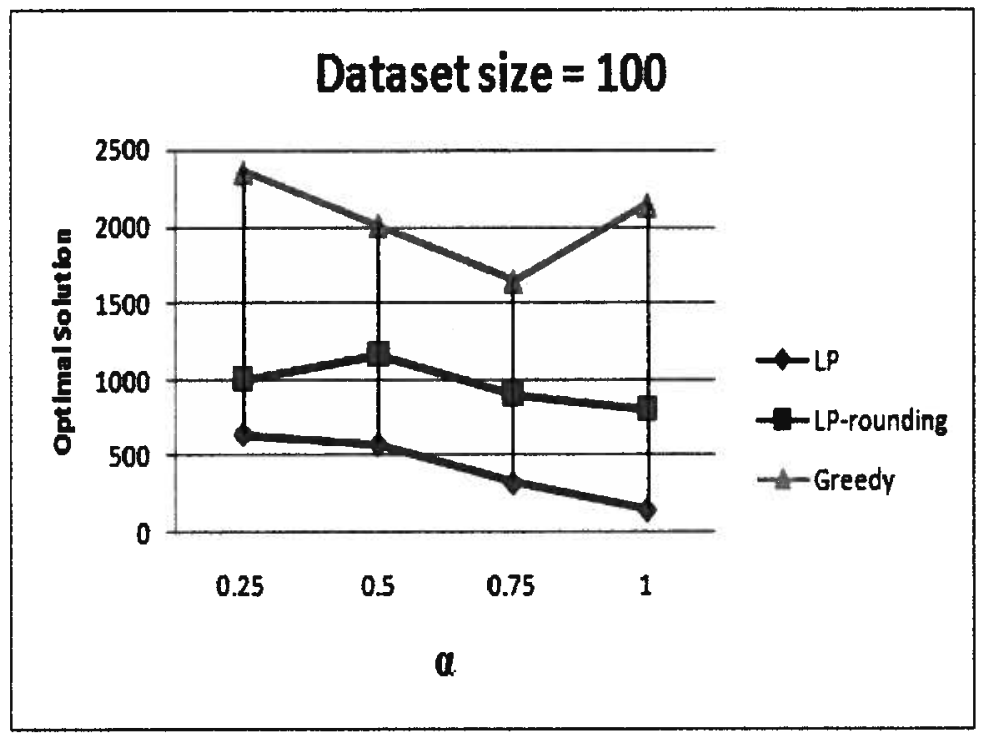

Figure 3.3: Performance of different algorithms on dataset of size 100 .

reason is that all users start with equal initial credits.

\subsection{Conclusion}

In this chapter, we consider exchange market problems over time and study efficient mechanisms that improve the users' waiting times and also give incentive to users to be more active by maximizing fairness in the system. In order to achieve the above objectives, we introduce points and credits to the system and we show that waiting times would improve by using points. Moreover, we show that the problem of fairness maximization not only is NP-complete, but also inapproximable. Therefore, we propose two heuristic algorithms: linear programming-based and a greedy algorithm. We perform experiments to examine the performance and scalability of the proposed 
Chapter 3. Online Exchange Markets Over Time

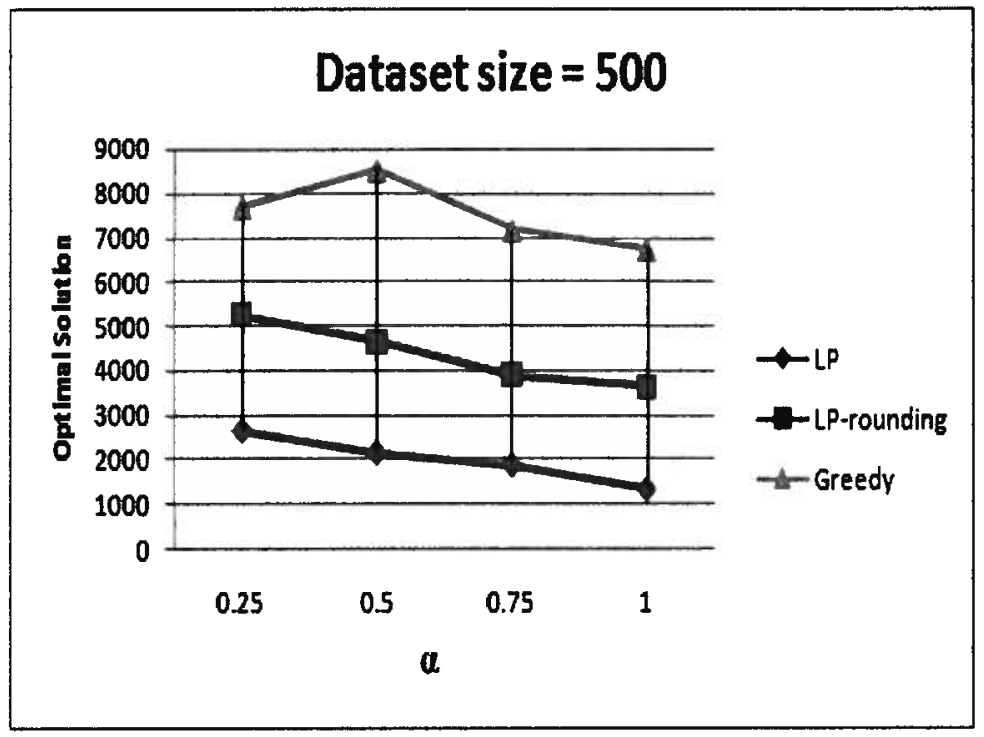

Figure 3.4: Performance of different algorithms on dataset of size 500 .

algorithms. The experiments show that both heuristics perform much better than the worst-case performance. Moreover, LP-rounding performs better, however the greedy heuristic is much faster. 
Chapter 3. Online Exchange Markets Over Time

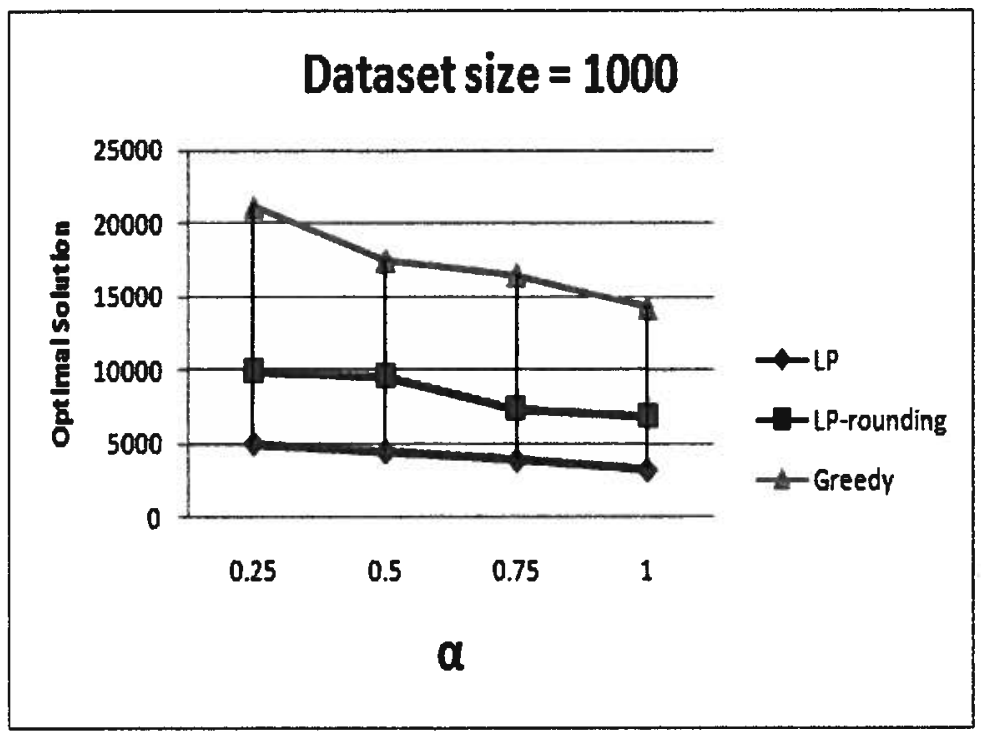

Figure 3.5: Performance of different algorithms on dataset of size 1000.

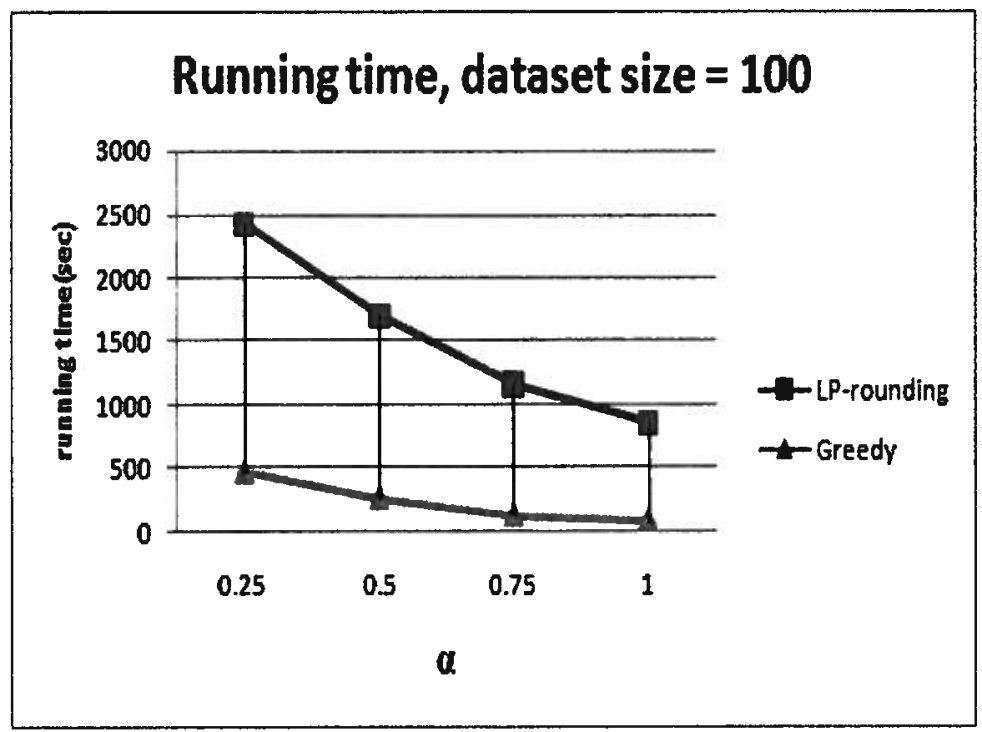

Figure 3.6: Running times for dataset of size 100 . 


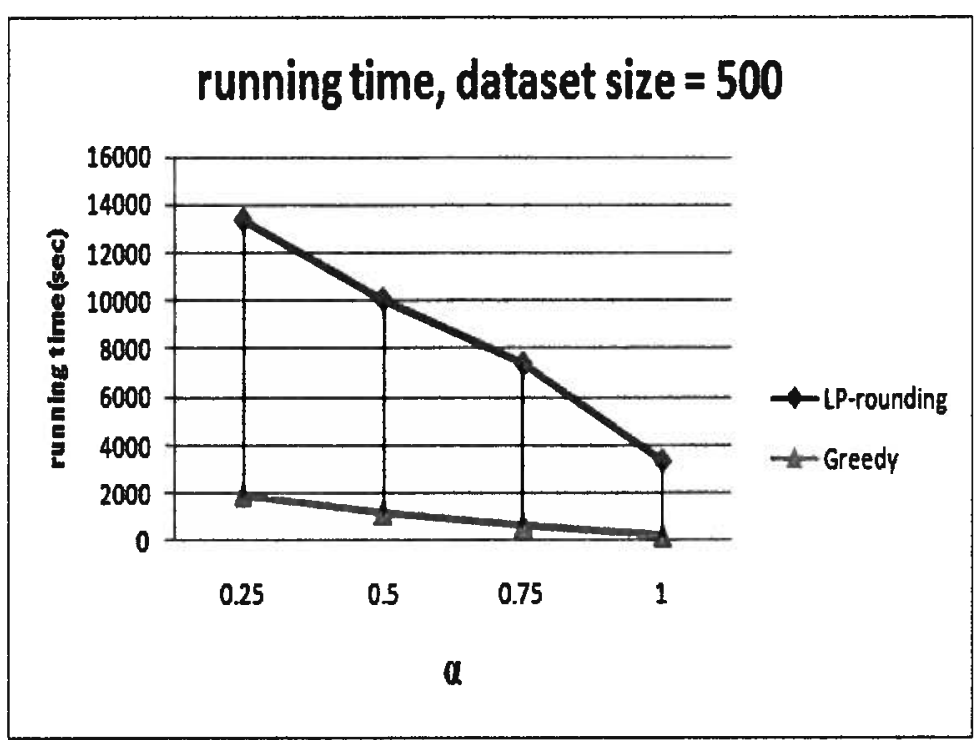

Figure 3.7: Running times for dataset of size 500 .

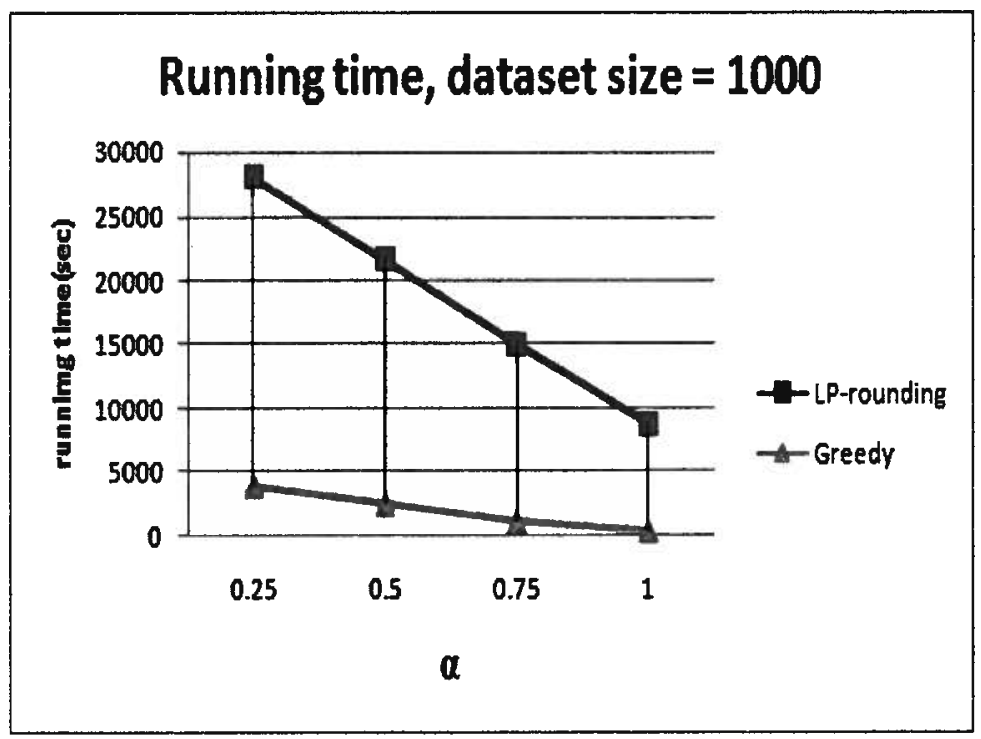

Figure 3.8: Running times for dataset of size 1000 . 


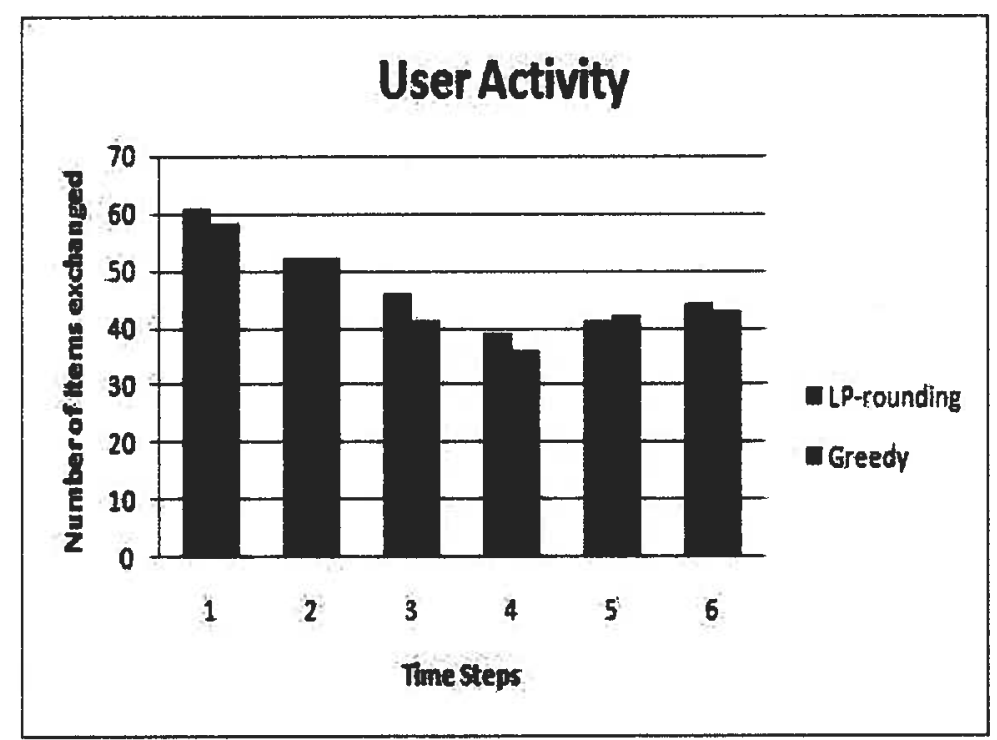

Figure 3.9: Number of transacting users in 6 consecutive time windows. 


\section{Chapter 4}

\section{Conclusions and Future}

\section{Work}

In this thesis, we motivate the use of online exchange markets as an application over social networks and propose specific market exchange problems where users own item lists and have a list of wishes and can exchange items with one another. For these problems, we propose a few optimization problems, study the complexity of these problems, propose various algorithms with guaranteed approximation factor for some of these problems, and finally perform experimental results to evaluate the performance of several approximation and heuristic algorithms.

In the one-shot deterministic case, we study simple market exchanges in which the only acceptable exchanges are in the form of swaps. While for a similar well-studied problem called the kidney exchange problem, this problem is solvable in polynomial time, we prove that this is NP-complete in our case. When longer exchange cycles are allowed, kidney exchange was shown to be NP-complete [5] and this extends to our setting. We also propose a probabilistic exchange market problem in which there are probabilities associated with a user engaging with another in an exchange. The hardness 
results extend to this case. By leveraging a close connection to the weighted $k$-set packing problem, we develop a heuristic algorithm (Maximal) and approximation algorithms (Greedy, Local Search, and Greedy/Local Search). Using this connection, we show that known approximation bounds for these algorithms for weighted $k$-set packing carry over to our exchange market problems both deterministic and probabilistic. We conduct a detailed experimentation using synthetically generated data sets, comparing different algorithms on output quality and running time and studying the extent of increase in coverage as maximum cycle length is increased, as well as the impact of data skew on coverage. Our results show that while Maximal does not have provable approximation guarantee, its performance in practice may be comparable to that of the other algorithms. This is interesting given that Maximal is by far the most efficient.

Finally, we consider similar exchange market problems over time and study efficient mechanisms that improve the users' waiting times and also give incentive to users to be more active by maximizing fairness in the system. In order to achieve the above objectives, we introduce points and credits to the system and we show that waiting times would improve by using points. Moreover, we show that the problem of fairness maximization not only is NP-complete, but also inapproximable. Therefore, we propose two heuristic algorithms: linear programming-based and a greedy algorithm. We perform experiments to examine the performance and scalability of the proposed algorithms. The experiments show that both heuristics perform much better than the worst-case performance. Moreover, LP-rounding performs better, however the greedy heuristic is much faster. 
Chapter 4. Conclusions and Future Work

As a future work, further investigation of these algorithms on real data sets would be valuable. Designing improved approximation algorithms or proving some stronger inapproximability results for the optimization problems discussed in this thesis are desirable. Also, it is interesting to study special variants of these problems that appear in practice, and design approximation algorithms with provably improved approximation factor. Finally it would be interesting to study incentive issues in these settings, and design mechanisms that perform well in the presence of strategic users who may not reveal their true parameters (e.g., their item lists). In such settings, users may lie about their items lists to get a better assignment. To deal with such settings, one needs to design (truthful) mechanisms that give incentive to users to reveal their true parameters. It is also interesting to explore if the proposed mechanism converges to an equilibrium. 


\section{Bibliography}

[1] "http://blog.compete.com/2007/01/25/top-20-websites-ranked-bytime-spent/".

[2] http://www.oddshoe.org.

[3] http://www.peerflix.com.

[4] http://www.readitswapit.co.uk.

[5] David J. Abraham, Avrim Blum, and Tuomas Sandholm. Clearing algorithms for barter exchange markets: Enabling nationwide kidney exchanges. In ACM Conference on Electronic Commerce, pages 295304, June 13-16 2007.

[6] David J. Abraham, Ning Chen, Vijay Kumar, and Vahab Mirrokni. Assignment problems in rental markets. In WINE 2006, pages 198213, 2006.

[7] G. Adomavicius and A. Tuzhilin. Toward the next generation of recommender systems: A survey of the state-of-the-art and possible extensions. IEEE Transactions on Knowledge and Data Engineering, 17(6):734-749, 2005. 
[8] Esther M. Arkin and Refael Hassin. On local search for weighted k-set packing. In ESA 1997, pages 13-22, 1997.

[9] M. Blaser and B. Siebert. Computing cycle covers without short cycles. In In Proceedings of the 34st Annual European Symposium of Algorithms, 2001.

[10] Barun Chandra and Magnus Halldorsson. Greedy local improvement and weighted set packing approximation. In SODA 1999, pages 169$176,1999$.

[11] W.W. Cohen, R.E. Schapire, and Y. Singer. Learning to order things. Journal of Artificial Intelligence Research, 10:243-270, 1999.

[12] J. Edmonds and E. Johnson. Matching euler tours and the chinese postman problem. Mathematical programming, 5:88-124, 1973.

[13] Ozlem Ergun, Gultekin Kuyzu, and Martin Savelsbergh. Collaborative logistics: The shipper collaboration problem. Computers and Operations Research Odysseus, 2003.

[14] Y. Freund, R. Iyer, R.E. Schapire, and Y. Singer. An efficient boosting algorithm for combining preferences. In 15th International Conference on Machine Learning, 1998.

[15] M. R. Garey and D. S. Johnson. Computers and Intractability. Freeman, New York, 1979. 
[16] O. Goldschmidt, D. Hochbaum, C. Hurkens, and G. Yu. Approximation algorithms for the k-clique covering problem. SIAM Journal of Discrete Math., 6(3):492-509, 1996.

[17] M. Guan. Graphic programming using odd and even points. Chinese Mathematics, 1:273-277, 1962.

[18] D. Hochbaum and E. Olinick. The bounded cycle-cover problem. INFORMS Journal on Computing, 13(2):104-109, 2001.

[19] Dorit S. Hochbaum and David B. Shmoys. A polynomial approximation scheme for scheduling on uniform processors: Using the dual approximation approach. SIAM Journal of Computing, 17(3):539-551, 1988.

[20] Ian Holyer. The np-completeness of some edge-partition problems. SIAM Journal of Computing,, 10(4):713-717, November 1981.

[21] Ellis Horowitz and Sartaj Sahni. Exact and approximate algorithms for scheduling nonidentical processors. $J . A C M, 23(2): 317-327,1976$.

[22] N. Immorlica, V. S. Mirrokni, and M. Mahdian. Cycle cover with short cycles. In Symposium on Theoretical Aspects of Computer Science (STACS), 2005.

[23] D. B. Shmoys J. K. Lenstra and Eva Tardos. Approximation algorithms for scheduling unrelated parallel machines. Math. Program., 46(3):259$271,1990$. 
[24] K. Gallagher. J. Parsons, P. Ralph. Using viewing time to infer user preference in recommender systems. In AAAI Workshop in Semantic Web Personalization, July 2004.

[25] R. Jin, L. Si, and C. Zhai. Preference-based graphic models for collaborative filtering. In 19th Conference Uncertainty in Artificial Intelligence (UAI 2003), August 2003.

[26] R. Jin, L. Si, C. Zhai, and J. Callan. Collaborative filtering with decoupled models for preferences and ratings. In 12th International Conference Information and Knowledge Management (CIKM 2003), Novemeber 2003.

[27] S. Lam and R. Sethi. Worst case analysis of two scheduling algorithms. SIAM Journal of Computing, 6(3):518-536, 1977.

[28] B.P.S. Murthi and S. Sarkar. The role of the management sciences in research on personalization. Management Science, 49(10):1344-1362, 2003.

[29] M. E. J. Newman. The structure and function of complex networks. SIAM Reviews, 45(2):167-256, 2003.

[30] Christos H. Papadimitriou. On the complexity of edge traversing. Journal of the $A C M, 23(3): 544-554,1976$.

[31] M.J.D. Powell. Approximation Theory and Methods. Cambridge University Press, 1981. 
[32] et al. R. Andersen. Trust-based recommendation systems: an axiomatic approach. 17th International World Wide Web Conference (WWW), 2008.

[33] B. Rachavachari and J. Veerasamy. A 3/2-approximation algorithm for the mixed postman problem. SIAM journal of Discrete Math., 12:425433, 1999.

[34] P. Resnick, N. Iakovou, M. Sushak, P. Bergstrom, and J. Riedl. Grouplens: An open architecture for collaborative filtering of netnews. In Computer Supported Cooperative Work Conference, 1994.

[35] P. Resnick, N. Iakovou, M. Sushak, P. Bergstrom, and J. Riedl. Grouplens: An open architecture for collaborative filtering of netnews. In Computer Supported Cooperative Work Conference, 1994.

[36] E. Rich. User modeling via stereotypes. Cognitive Science, 3(4):329$354,1979$.

[37] G. Salton. Automatic Text Processing. Addison-Wesley, 1989.

[38] G. Shani, R. Brafman, and D. Heckerman. An mdp-based recommender system. In 18th Conference Uncertainty in Artificial Intelligence, August, 2002.

[39] Carsten Thomassen. On the complexity of finding a minimum cycle cover of a graph. SIAM J. Comput., 26(3):675-677, 1997.

[40] F. Wu and L. Zhang. Proportional response dynamics leads to market equilibrium. In STOC, pages $354-363,2007$. 


\section{Bibliography}

[41] C.Q. Zhang. Integer flows and cycle cover of graphs. Marcel Dekker, 1997. 